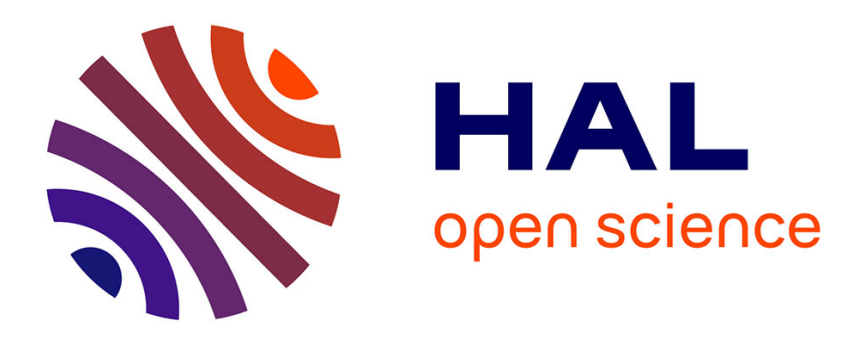

\title{
Transport of Charged Particles Under Fast Oscillating Magnetic Fields
}

\author{
Mihai Bostan
}

\section{To cite this version:}

Mihai Bostan. Transport of Charged Particles Under Fast Oscillating Magnetic Fields. SIAM Journal on Mathematical Analysis, 2012, 44 (3), pp.1415-1447. 10.1137/100797400 . hal-01266545

\section{HAL Id: hal-01266545 \\ https://hal.science/hal-01266545}

Submitted on 2 Feb 2016

HAL is a multi-disciplinary open access archive for the deposit and dissemination of scientific research documents, whether they are published or not. The documents may come from teaching and research institutions in France or abroad, or from public or private research centers.
L'archive ouverte pluridisciplinaire HAL, est destinée au dépôt et à la diffusion de documents scientifiques de niveau recherche, publiés ou non, émanant des établissements d'enseignement et de recherche français ou étrangers, des laboratoires publics ou privés. 


\title{
Transport of charged particles under fast oscillating magnetic fields
}

\author{
Mihai Bostan *
}

(March 18, 2012)

\begin{abstract}
The energy production through thermo-nuclear fusion requires the confinement of the plasma into a bounded domain. In most of the cases, such configurations are obtained by using strong magnetic fields. Several models exist for describing the evolution of a strongly magnetized plasma, i.e., guiding-center approximation, finite Larmor radius regime, etc. The topic of this paper concerns a different approach leading to plasma confinement. More exactly we are interested in mathematical models with fast oscillating magnetic fields. We provide rigorous derivations for this kind of models and analyze their properties.
\end{abstract}

Keywords: Vlasov equation, Average operator, Multi-scale analysis.

AMS classification: $35 \mathrm{Q} 75,78 \mathrm{~A} 35,82 \mathrm{D} 10$.

\section{Introduction}

Motivated by the energy production through thermo-nuclear fusion, many research programs concern plasma confinement models. It is well known that good confinement properties are obtained under strong magnetic fields $B^{\varepsilon}=\mathcal{O}(1 / \varepsilon)$ with $\varepsilon>0$ a small

*Laboratoire de Mathématiques de Besançon, UMR CNRS 6623, Université de Franche-Comté, 16 route de Gray, 25030 Besançon Cedex France. E-mail : mbostan@univ-fcomte.fr 
parameter. Using the kinetic description and neglecting the particle collisions lead to the Vlasov equation

$$
\partial_{t} f^{\varepsilon}+\frac{p}{m} \cdot \nabla_{x} f^{\varepsilon}+e\left(E^{\varepsilon}(t, x)+\frac{p}{m} \wedge B^{\varepsilon}(t, x)\right) \cdot \nabla_{p} f^{\varepsilon}=0, \quad(t, x, p) \in \mathbb{R}_{+} \times \mathbb{R}^{3} \times \mathbb{R}^{3}
$$

with the initial condition

$$
f^{\varepsilon}(0, x, p)=f^{\text {in }}(x, p), \quad(x, p) \in \mathbb{R}^{3} \times \mathbb{R}^{3} .
$$

Here $f^{\varepsilon}=f^{\varepsilon}(t, x, p) \geq 0$ is the distribution function of the particles in the positionmomentum phase space $(x, p) \in \mathbb{R}^{3} \times \mathbb{R}^{3}, m$ is the particle mass, $e$ is the particle charge and $\left(E^{\varepsilon}, B^{\varepsilon}\right)$ stands for the electro-magnetic field.

Standard configurations ensuring confinement are those obtained by applying strong magnetic fields. For example, assuming that the electric field derives from a given potential $E=-\nabla_{x} \phi$ and the magnetic field is stationary, divergence free

$$
B^{\varepsilon}(x)=\frac{B(x)}{\varepsilon} b(x), \operatorname{div}_{x}(B b)=0, \quad 0<\varepsilon<<1
$$

for some scalar positive function $B(x)$ and some field of unitary vectors $b(x)$, lead to the Vlasov equation

$$
\partial_{t} f^{\varepsilon}+\frac{p}{m} \cdot \nabla_{x} f^{\varepsilon}+\left(e E(t, x)+\frac{\omega_{c}(x)}{\varepsilon} p \wedge b(x)\right) \cdot \nabla_{p} f^{\varepsilon}=0, \omega_{c}(x)=\frac{e B(x)}{m}
$$

whose limit as $\varepsilon \searrow 0$ is known as the guiding-center approximation. The particles rotate around the magnetic lines and the radius of this circular motion, which is called the Larmor radius $\rho_{L}$, is proportional to the inverse of the magnetic field. Therefore when the magnetic field is strong, the typical Larmor radius vanishes and the particles remain confined along the magnetic lines. But the frequency of these rotations, which is called the cyclotronic frequency, is proportional with the magnetic field. Consequently, high magnetic fields introduce small time scales, since the cyclotronic period is much smaller than the observation time. Clearly, the transport equation (3) involves multiple scales: fast motion around the magnetic lines driven by the Laplace force in $\frac{\omega_{c}(x)}{\varepsilon}(p \wedge$ b) $\cdot \nabla_{p}$ and slow motion corresponding to the advection $\frac{p}{m} \cdot \nabla_{x}+e E \cdot \nabla_{p}$.

From the numerical point of view, the efficient resolution of (3) requires multiple scale analysis [3] or homogenization techniques. It is also possible to appeal to Lagrangian and Hamiltonian methods [9]. For a unified treatment of the main physical 
ideas and theoretical methods that have emerged on magnetic plasma confinement we refer to $[15]$.

The guiding-center approximation for the Vlasov-Maxwell system was studied in [5] by the modulated energy method. The case of three dimensional general magnetic shapes (3) has been studied in [7], using a general method, based on ergodicity, introduced in [6]. It was proved in [7] that the limit density $f=\lim _{\varepsilon \backslash 0} f^{\varepsilon}$ satisfies the Vlasov equation

$$
\partial_{t} f+b(x) \otimes b(x) \frac{p}{m} \cdot \nabla_{x} f+(e b(x) \otimes b(x) E+\omega(x, p) \tilde{p}) \cdot \nabla_{p} f=0
$$

where for any $(x, p)$ with $p \wedge b(x) \neq 0$ the symbol $\tilde{p}$ stands for the orthogonal momentum to $p$, contained in the plane determined by $b(x)$ and $p$, and such that its coordinate along $b(x)$ is positive, that means

$$
\tilde{p}=|p \wedge b(x)| b(x)-(p \cdot b(x)) \frac{b(x) \wedge(p \wedge b(x))}{|p \wedge b(x)|}
$$

and the frequency $\omega(x, p)$ is given by

$$
\omega(x, p)=\frac{|p \wedge b(x)|}{2 m} \operatorname{div}_{x} b-\frac{(p \cdot b(x))}{m}\left(\frac{\partial b}{\partial x} b(x) \cdot \frac{p}{|p \wedge b(x)|}\right), p \wedge b(x) \neq 0 .
$$

The analysis of the Vlasov or Vlasov-Poisson equations with large external magnetic field have been carried out in [10], [12], [8], [11], [13]. The numerical approximation of the gyrokinetic models has been performed in [14] using semi-Lagrangian schemes. Other methods are based on the water bag representation of the distribution function [16].

Notice that configurations with large magnetic field amplitude require huge energy since the magnetic energy is quadratic with respect to $\left|B^{\varepsilon}\right|=B / \varepsilon$.

We investigate here models with fast oscillating magnetic fields

$$
B^{\varepsilon}(t, x)=\theta(t / \varepsilon) B(x) b(x), \quad 0<\varepsilon<<1
$$

where $\theta=\theta(s)$ is a given $T$ periodic profile of class $C^{1}$. The magnetic energy dissipated in this case is much lower than for the guiding-center approximation and remains of order of $|B|^{2}$. Therefore such models will be more interesting for real life applications, provided they still have good confinement properties. At this stage we neglect the gradient and curvature effects of the magnetic field, assuming that

$$
B^{\varepsilon}=B^{\varepsilon}(t)=\theta(t / \varepsilon)(0,0, B)
$$


for some constant $B>0$. The general model including gradient and curvature effects will be discussed in Section 7. The vector potential corresponding to this magnetic field, i.e., satisfying $B^{\varepsilon}=\operatorname{curl}_{x} A^{\varepsilon}$, $\operatorname{div}_{x} A^{\varepsilon}=0$ is given by

$$
A^{\varepsilon}(t, x)=-\frac{B}{2} \theta(t / \varepsilon)^{\perp} x,{ }^{\perp} x=\left(x_{2},-x_{1}, 0\right) .
$$

Decomposing the electric field into gradient and rotational parts

$$
E^{\varepsilon}=-\nabla_{x} \phi+\operatorname{curl}_{x} \psi^{\varepsilon}
$$

we deduce, by Faraday's law $\partial_{t} B^{\varepsilon}+\operatorname{curl}_{x} E^{\varepsilon}=0$ that

$$
\operatorname{curl}_{x}\left(\partial_{t} A^{\varepsilon}+\operatorname{curl}_{x} \psi^{\varepsilon}\right)=0, \operatorname{div}_{x}\left(\partial_{t} A^{\varepsilon}+\operatorname{curl}_{x} \psi^{\varepsilon}\right)=0
$$

and therefore the electric field induced by the time fluctuations of the magnetic field is

$$
\operatorname{curl}_{x} \psi^{\varepsilon}=-\partial_{t} A^{\varepsilon}=\frac{B}{2 \varepsilon} \theta^{\prime}(t / \varepsilon)^{\perp} x .
$$

The Vlasov equation becomes, with the notations ${ }^{\perp} p=\left(p_{2},-p_{1}, 0\right)$ and $E=-\nabla_{x} \phi$

$$
\partial_{t} f^{\varepsilon}+\frac{p}{m} \cdot \nabla_{x} f^{\varepsilon}+\left(e E(t, x)+\frac{m \omega_{c}}{2 \varepsilon} \theta^{\prime}(t / \varepsilon)^{\perp} x+\omega_{c} \theta(t / \varepsilon)^{\perp} p\right) \cdot \nabla_{p} f^{\varepsilon}=0 .
$$

Here $E=-\nabla_{x} \phi$ is a given irrotational electric field or can be determined eventually by solving the Poisson equation

$$
\operatorname{div}_{x} E(t)=-\Delta_{x} \phi(t)=\frac{e}{\varepsilon_{0}}\left\{\int_{\mathbb{R}^{3}} f^{\varepsilon}(t, x, p) \mathrm{d} p-n_{0}(x)\right\}, \quad t \in \mathbb{R}_{+}, \quad x \in \mathbb{R}^{3} .
$$

The concentration $n_{0}(x)$ corresponds to a neutralizing background of charged particles of opposite sign and $\varepsilon_{0}$ is the electric permittivity of the vacuum.

Our paper is organized as follows. The main results are presented in Section 2. In Section 3 we introduce the mathematical tools that we need for our analysis. It mainly concerns the average operator with respect to characteristic flows. We discuss its main properties as range characterization, Poincaré and Sobolev inequalities. Some commutation results are established in Section 4. Section 5 is devoted to the derivation of the limit model, which follows in a natural way by appealing to the average operator introduced before. We establish the conservation of the total energy and justify the confinement properties for such a model. The asymptotic behaviour towards this limit model is analyzed in Section 6. The general three dimensional setting is investigated in the last section. 


\section{Presentation of the model and main results}

The Vlasov equation (5) reduces to the characteristic system

$$
\frac{d X^{\varepsilon}}{d t}=\frac{P^{\varepsilon}(t)}{m}, \frac{d P^{\varepsilon}}{d t}=e E\left(t, X^{\varepsilon}(t)\right)+\frac{m \omega_{c}}{2 \varepsilon} \theta^{\prime}(t / \varepsilon){ }^{\perp} X^{\varepsilon}(t)+\omega_{c} \theta(t / \varepsilon){ }^{\perp} P^{\varepsilon}(t) .
$$

It is convenient to introduce the fast variable $s=t / \varepsilon$ and the standard ansatz

$$
X^{\varepsilon}(t)=X^{0}(t, t / \varepsilon)+\varepsilon X^{1}(t, t / \varepsilon)+\ldots, \quad P^{\varepsilon}(t)=P^{0}(t, t / \varepsilon)+\varepsilon P^{1}(t, t / \varepsilon)+\ldots
$$

(here all dependences with respect to the fast variable $s$ are supposed $T$ periodic, as the profile $\theta=\theta(s))$ leading to

$$
\partial_{t} X^{0}+\frac{1}{\varepsilon} \partial_{s} X^{0}+\varepsilon\left(\partial_{t} X^{1}+\frac{1}{\varepsilon} \partial_{s} X^{1}\right)+\ldots=\frac{P^{0}}{m}+\varepsilon \frac{P^{1}}{m}+\ldots
$$

and

$$
\begin{aligned}
\partial_{t} P^{0}+\frac{1}{\varepsilon} \partial_{s} P^{0}+\varepsilon\left(\partial_{t} P^{1}+\frac{1}{\varepsilon} \partial_{s} P^{1}\right)+\ldots & =e E\left(t, X^{0}+\varepsilon X^{1}+\ldots\right) \\
& +\frac{m \omega_{c}}{2 \varepsilon} \theta^{\prime}(t / \varepsilon)^{\perp}\left(X^{0}+\varepsilon X^{1}+\ldots\right) \\
& +\omega_{c} \theta(t / \varepsilon)^{\perp}\left(P^{0}+\varepsilon P^{1}+\ldots\right) .
\end{aligned}
$$

At least formally one gets the equations

$$
\partial_{s} X^{0}=0, \quad \partial_{s} P^{0}=\frac{m \omega_{c}}{2} \theta^{\prime}(s){ }^{\perp} X^{0}
$$

at the lowest order $\varepsilon^{-1}$ and

$$
\partial_{t} X^{0}+\partial_{s} X^{1}=\frac{P^{0}}{m}, \quad \partial_{t} P^{0}+\partial_{s} P^{1}=e E\left(t, X^{0}\right)+\frac{m \omega_{c}}{2} \theta^{\prime}(s){ }^{\perp} X^{1}+\omega_{c} \theta(s){ }^{\perp} P^{0}
$$

at the next order $\varepsilon^{0}$. It follows that the quantities

$$
X^{0}, \quad Q^{0}=P^{0}-\frac{m \omega_{c}}{2} \theta(s)^{\perp} X^{0}
$$

depend only on $t$. Therefore, in order to obtain the characteristic equations satisfied by the leading order terms $\left(X^{0}, P^{0}\right)$ we write the equations $(9)$ in terms of $\left(X^{0}, Q^{0}\right)$ and eliminate $\left(X^{1}, P^{1}\right)$ by averaging with respect to the fast variable $s$ over one period. The first equation in (9) becomes

$$
\partial_{t} X^{0}+\partial_{s} X^{1}=\frac{Q^{0}}{m}+\frac{\omega_{c}}{2} \theta(s)^{\perp} X^{0}
$$


and therefore averaging with respect to $s$ yields

$$
\frac{d X^{0}}{d t}=\frac{Q^{0}(t)}{m}+\frac{\omega_{c}}{2}\langle\theta\rangle{ }^{\perp} X^{0}(t),\langle\theta\rangle=\frac{1}{T} \int_{0}^{T} \theta(s) \mathrm{d} s .
$$

Similarly, the second equation in (9) implies

$$
\begin{aligned}
\partial_{t}\left(Q^{0}+\frac{m \omega_{c}}{2} \theta(s){ }^{\perp} X^{0}\right)+\partial_{s} P^{1} & =e E\left(t, X^{0}\right)+\frac{m \omega_{c}}{2} \partial_{s}\left\{\theta{ }^{\perp} X^{1}\right\}-\frac{m \omega_{c}}{2} \theta(s) \partial_{s}{ }^{\perp} X^{1} \\
& +\omega_{c} \theta(s)^{\perp}\left\{Q^{0}+\frac{m \omega_{c}}{2} \theta(s)^{\perp} X^{0}\right\} \\
& =e E\left(t, X^{0}\right)+\frac{m \omega_{c}}{2} \partial_{s}\left\{\theta^{\perp} X^{1}\right\} \\
& -\frac{m \omega_{c}}{2} \theta(s) \perp\left\{\frac{Q^{0}}{m}+\frac{\omega_{c}}{2} \theta(s)^{\perp} X^{0}-\partial_{t} X^{0}\right\} \\
& +\omega_{c} \theta(s){ }^{\perp} Q^{0}+\frac{m \omega_{c}^{2}}{2} \theta^{2}(s){ }^{\perp \perp} X^{0} .
\end{aligned}
$$

Finally one gets

$$
\partial_{t} Q^{0}+\partial_{s} P^{1}=e E\left(t, X^{0}\right)+\frac{m \omega_{c}}{2} \partial_{s}\left\{\theta^{\perp} X^{1}\right\}+\frac{\omega_{c}}{2} \theta(s){ }^{\perp} Q^{0}+\frac{m \omega_{c}^{2}}{4} \theta^{2}(s){ }^{\perp \perp} X^{0}
$$

and therefore, averaging with respect to $s$ yields

$$
\frac{d Q^{0}}{d t}=e E\left(t, X^{0}(t)\right)+\frac{\omega_{c}}{2}\langle\theta\rangle{ }^{\perp} Q^{0}(t)+\frac{m \omega_{c}^{2}}{4}\left\langle\theta^{2}\right\rangle{ }^{\perp}{ }^{\perp} X^{0}(t), \quad\left\langle\theta^{2}\right\rangle=\frac{1}{T} \int_{0}^{T} \theta^{2}(s) \mathrm{d} s .
$$

We associate to the characteristic equations (10), (11) the transport equation

$$
\partial_{t} g^{0}+\left(\frac{q}{m}+\frac{\omega_{c}}{2}\langle\theta\rangle^{\perp} x\right) \cdot \nabla_{x} g^{0}+\left(e E(t, x)+\frac{\omega_{c}}{2}\langle\theta\rangle{ }^{\perp} q+\frac{m \omega_{c}^{2}}{4}\left\langle\theta^{2}\right\rangle{ }^{\perp \perp} x\right) \cdot \nabla_{q} g^{0}=0 .
$$

Since we have

$$
\left(X^{\varepsilon}(t), P^{\varepsilon}(t)\right) \approx\left(X^{0}(t, t / \varepsilon), P^{0}(t, t / \varepsilon)\right)=\left(X^{0}(t), Q^{0}(t)+\frac{m \omega_{c}}{2} \theta(t / \varepsilon)^{\perp} X^{0}(t)\right)
$$

and assuming that $X^{\varepsilon}(0)=x, P^{\varepsilon}(0)=p$ we can write

$$
\begin{gathered}
f^{\varepsilon}\left(t, X^{0}(t, t / \varepsilon), P^{0}(t, t / \varepsilon)\right) \approx f^{\varepsilon}\left(t, X^{\varepsilon}(t), P^{\varepsilon}(t)\right)=f^{\mathrm{in}}(x, p) \\
g^{0}\left(t, X^{0}(t, t / \varepsilon), P^{0}(t, t / \varepsilon)-\frac{m \omega_{c}}{2} \theta(t / \varepsilon){ }^{\perp} X^{0}(t, t / \varepsilon)\right)=g^{0}\left(t, X^{0}(t), Q^{0}(t)\right)=g^{\mathrm{in}}(x, q)
\end{gathered}
$$

and therefore we can expect that

$$
f^{\varepsilon}(t, x, p) \approx g^{0}\left(t, x, q=p-\frac{m \omega_{c}}{2} \theta(t / \varepsilon)^{\perp} x\right), \text { as } \varepsilon \searrow 0
$$

provided that the initial conditions are well prepared. Introducing the density $f^{0}(t, s, x, p)=$ $g^{0}\left(t, x, p-m \omega_{c} \theta(s) / 2^{\perp} x\right)$ in the phase space $(s, x, p)$ we deduce that $f^{\varepsilon}(t, x, p) \approx$ $f^{0}(t, t / \varepsilon, x, p)$ as $\varepsilon \searrow 0$. 
Theorem 2.1 Assume that $E \in L_{\text {loc }}^{1}\left(\mathbb{R}_{+} ; L^{\infty}\left(\mathbb{R}^{3}\right)\right), f^{\text {in }} \in L^{2}\left(\mathbb{R}^{3} \times \mathbb{R}^{3}\right)$. For any $\varepsilon>0$ let $f^{\varepsilon} \in L^{\infty}\left(\mathbb{R}_{+} ; L^{2}\left(\mathbb{R}_{x}^{3} \times \mathbb{R}_{p}^{3}\right)\right)$ be a weak solution of $(5)$. Then there is a sequence $\left(\varepsilon_{n}\right)_{n}$ converging to zero such that $\left(f^{\varepsilon_{n}}\right)_{n}$ two-scale converges towards a weak solution of

$$
\begin{gathered}
\partial_{t} f^{0}+\left(\frac{p}{m}-\frac{\omega_{c}}{2}(\theta(s)-\langle\theta\rangle)^{\perp} x\right) \cdot \nabla_{x} f^{0} \\
+\left(e E(t, x)+\frac{\omega_{c}}{2}(\theta(s)+\langle\theta\rangle)^{\perp} p+\frac{m \omega_{c}^{2}}{4}\left(\left\langle\theta^{2}\right\rangle-\theta^{2}(s){ }^{\perp \perp} x\right) \cdot \nabla_{p} f^{0}=0\right. \\
f^{0}(0, s, x, p)=f^{\text {in }}\left(x, p-\frac{m \omega_{c}}{2}(\theta(s)-\theta(0))^{\perp} x\right) \in \operatorname{ker} \mathcal{T} .
\end{gathered}
$$

Consequently we have to study the confinement properties of the limit model (12) (or (13)). Indeed, such models lead to confinement. For convincing ourselves let us consider a particular case, that of vanishing electric potential $\phi=0$. The characteristic system for $\left(X^{0}, Q^{0}\right)$ becomes

$$
\frac{d X^{0}}{d t}=\frac{Q^{0}(t)}{m}+\frac{\omega_{c}}{2}\langle\theta\rangle{ }^{\perp} X^{0}(t), \quad \frac{d Q^{0}}{d t}=\frac{\omega_{c}}{2}\langle\theta\rangle{ }^{\perp} Q^{0}(t)+\frac{m \omega_{c}^{2}}{4}\left\langle\theta^{2}\right\rangle{ }^{\perp \perp} X^{0}(t)
$$

implying that

$$
\frac{d^{2} X^{0}}{d t^{2}}-\frac{\omega_{c}^{2}}{4}\left(\left\langle\theta^{2}\right\rangle-\langle\theta\rangle^{2}\right)^{\perp \perp} X^{0}(t)=\omega_{c}\langle\theta\rangle \frac{d^{\perp} X^{0}}{d t} .
$$

Multiplying by $\frac{d X^{0}}{d t}$ we obtain the conservation

$$
\frac{d}{d t}\left\{\frac{1}{2}\left|\frac{d X^{0}}{d t}\right|^{2}+\left.\left.\frac{\omega_{c}^{2}}{4}\left(\left\langle\theta^{2}\right\rangle-\langle\theta\rangle^{2}\right) \frac{1}{2}\right|^{\perp \perp} X^{0}\right|^{2}\right\}=0 .
$$

If $\theta$ is not a constant profile (i.e., the magnetic field oscillates in time), then

$$
\frac{\omega_{c}^{2}}{4}\left(\left\langle\theta^{2}\right\rangle-\langle\theta\rangle^{2}\right)=\frac{\omega_{c}^{2}}{4}\left\langle(\theta-\langle\theta\rangle)^{2}\right\rangle>0
$$

and clearly the projection of $X^{0}(t)$ on the orthogonal directions with respect to the magnetic field oscillates around the magnetic lines. The oscillation frequencies can be computed explicitely in this particular case. Observe that the components $\left(X_{1}, X_{2}\right)$ satisfy

$$
X_{j}^{(4)}+\frac{\omega_{c}^{2}}{2}\left(\left\langle\theta^{2}\right\rangle+\langle\theta\rangle^{2}\right) X_{j}^{(2)}+\frac{\omega_{c}^{4}}{16}\left(\left\langle\theta^{2}\right\rangle-\langle\theta\rangle^{2}\right)^{2} X_{j}=0 .
$$

The roots of the characteristic polynomial are purely imaginary

$$
\pm i \frac{\omega_{c}}{2}\left(\sqrt{\left\langle\theta^{2}\right\rangle} \pm\langle\theta\rangle\right)
$$


and therefore the oscillation frequencies in the plane $\left(x_{1}, x_{2}\right)$ are $\frac{\omega_{c}}{2}\left(\sqrt{\left\langle\theta^{2}\right\rangle} \pm\langle\theta\rangle\right)$. The plasma remains confined along the magnetic lines. Generally we establish the following result

Theorem 2.2 Assume that $\lambda \in C^{1}(\mathbb{R})$ is nonincreasing, nonnegative and vanishes on $\left[L,+\infty\left[\right.\right.$, for some $L>0$. Let the initial condition $f^{\text {in }}$ satisfy

$$
f^{\text {in }}(x, p) \leq \lambda\left(\chi\left(x, p-m \omega_{c} \theta(0) / 2^{\perp} x\right)+e \phi(0, x)\right)
$$

where

$$
\chi(x, q)=\frac{1}{2 m}\left|q+\frac{m \omega_{c}}{2}\langle\theta\rangle{ }^{\perp} x\right|^{2}+\frac{m \omega_{c}^{2}}{4}\left(\left\langle\theta^{2}\right\rangle-\langle\theta\rangle^{2}\right) \frac{\left.\left.\right|^{\perp} x\right|^{2}}{2} .
$$

If the electric potential $\phi \in C^{1}\left(\mathbb{R}_{+} \times \mathbb{R}^{3}\right)$ satisfies

$$
\lim _{\left.\right|^{\perp} x \mid \rightarrow+\infty}\left\{e \phi(t, x)-\int_{0}^{t} \sup _{y \in \mathbb{R}^{3}}\left\{e \partial_{t} \phi(s, y)\right\} \mathrm{d} s+\frac{m \omega_{c}^{2}}{4}\left(\left\langle\theta^{2}\right\rangle-\langle\theta\rangle^{2}\right) \frac{\left.\left.\right|^{\perp} x\right|^{2}}{2}\right\}=+\infty
$$

uniformly with respect to $t \in \mathbb{R}_{+}, x_{3} \in \mathbb{R}$, then there is a constant $R>0$ such that for any $t \in \mathbb{R}_{+}, s \in \mathbb{R}$ the solution of the problem (13), (14) verifies

$$
\operatorname{supp} f^{0}(t, s, \cdot, \cdot) \subset\left\{(x, p):\left.\right|^{\perp} x \mid \leq R\right\}
$$

We also prove a strong convergence result

Theorem 2.3 Assume that $E \in L_{\mathrm{loc}}^{1}\left(\mathbb{R}_{+} ; W^{2, \infty}\left(\mathbb{R}^{3}\right)\right), \partial_{t} E \in L_{\mathrm{loc}}^{1}\left(\mathbb{R}_{+} ; L^{\infty}\left(\mathbb{R}^{3}\right)\right)$, the initial condition $f^{\text {in }}$ has compact support and belongs to $W^{2, \infty}\left(\mathbb{R}^{3} \times \mathbb{R}^{3}\right)$. Let $f^{0}(t, s, x, p)$ be the solution of (13), (14) and $\left(f^{\varepsilon}\right)_{\varepsilon}$ the solutions of the problems (5), (2). Then for any interval $[0, I] \subset \mathbb{R}_{+}$there is a constant $C(I)$ such that

$$
\left\|f^{\varepsilon}(t, \cdot, \cdot)-f^{0}(t, t / \varepsilon, \cdot, \cdot)\right\|_{L^{2}\left(\mathbb{R}^{3} \times \mathbb{R}^{3}\right)} \leq C(I) \varepsilon, \quad t \in[0, I], \varepsilon>0 .
$$

\section{Average operator}

The previous considerations clearly show that the limit of the Vlasov equation with fast oscillating magnetic field deals with multi-scale techniques and homogenization arguments. For the rigorous derivation of the limit model (12) we appeal to a slightly different method, based on Hilbert expansion at the density level

$$
f^{\varepsilon}(t, x, p)=f^{0}(t, t / \varepsilon, x, p)+\varepsilon f^{1}(t, t / \varepsilon, x, p)+\ldots
$$


In this section we assume that $E=-\nabla_{x} \phi$ is a given electric field. Plugging this ansatz into (5) leads to

$$
\begin{gathered}
\partial_{t} f^{0}+\frac{1}{\varepsilon} \partial_{s} f^{0}+\varepsilon\left(\partial_{t} f^{1}+\frac{1}{\varepsilon} \partial_{s} f^{1}\right)+\ldots+\frac{p}{m} \cdot\left(\nabla_{x} f^{0}+\varepsilon \nabla_{x} f^{1}+\ldots\right) \\
+\left(e E(t, x)+\frac{m \omega_{c}}{2 \varepsilon} \theta^{\prime}(t / \varepsilon)^{\perp} x+\omega_{c} \theta(t / \varepsilon){ }^{\perp} p\right) \cdot\left(\nabla_{p} f^{0}+\varepsilon \nabla_{p} f^{1}+\ldots\right)=0
\end{gathered}
$$

and we obtain formally

$$
\partial_{s} f^{0}+\frac{m \omega_{c}}{2} \theta^{\prime}(s)^{\perp} x \cdot \nabla_{p} f^{0}=0
$$

at the lowest order $\varepsilon^{-1}$ and

$$
\partial_{t} f^{0}+\frac{p}{m} \cdot \nabla_{x} f^{0}+\left(e E(t, x)+\omega_{c} \theta(s){ }^{\perp} p\right) \cdot \nabla_{p} f^{0}+\partial_{s} f^{1}+\frac{m \omega_{c}}{2} \theta^{\prime}(s){ }^{\perp} x \cdot \nabla_{p} f^{1}=0
$$

at the next order $\varepsilon^{0}$. The following operator will play a crucial role in our analysis

$$
\mathcal{T} u=\operatorname{div}_{(s, p)}\left\{u\left(1, \frac{m \omega_{c}}{2} \theta^{\prime}(s)^{\perp} x\right)\right\}
$$

with domain

$D(\mathcal{T})=\left\{u \in L_{\#}^{2}\left(\mathbb{R}_{s} ; L^{2}\left(\mathbb{R}_{x}^{3} \times \mathbb{R}_{p}^{3}\right)\right): \operatorname{div}_{(s, p)}\left\{u\left(1, \frac{m \omega_{c}}{2} \theta^{\prime}(s){ }^{\perp} x\right)\right\} \in L_{\#}^{2}\left(\mathbb{R}_{s} ; L^{2}\left(\mathbb{R}_{x}^{3} \times \mathbb{R}_{p}^{3}\right)\right)\right\}$

with $L_{\#}^{2}\left(\mathbb{R}_{s} ; X\right)$ the space of square integrable $T$ periodic functions $u: \mathbb{R} \rightarrow\left(X,\|\cdot\|_{X}\right)$, endowed with the norm

$$
\left(\int_{0}^{T}\|u(s)\|_{X}^{2} \mathrm{~d} s\right)^{1 / 2} .
$$

The notation $\|\cdot\|$ stands for the standard norm of $L_{\#}^{2}\left(\mathbb{R}_{s} ; L^{2}\left(\mathbb{R}_{x}^{3} \times \mathbb{R}_{p}^{3}\right)\right)$

$$
\|u\|=\left(\int_{0}^{T} \int_{\mathbb{R}^{3}} \int_{\mathbb{R}^{3}}|u(s, x, p)|^{2} \mathrm{~d} p \mathrm{~d} x \mathrm{~d} s\right)^{1 / 2} .
$$

We denote by $(S, X, P)=(S, X, P)(\tau ; s, x, p)$ the characteristics of the first order differential operator $\partial_{s}+\frac{m \omega_{c}}{2} \theta^{\prime}(s){ }^{\perp} x \cdot \nabla_{p}$

$$
\frac{d S}{d \tau}=1, \quad \frac{d X}{d \tau}=0, \quad \frac{d P}{d \tau}=\frac{m \omega_{c}}{2} \theta^{\prime}(S(\tau))^{\perp} X(S(\tau))
$$

with the conditions

$$
S(0 ; s, x, p)=s, \quad X(0 ; s, x, p)=x, \quad P(0 ; s, x, p)=p \text {. }
$$


It is easily seen that

$$
S(\tau ; s, x, p)=s+\tau, \quad X(\tau ; s, x, p)=x, \quad P(\tau ; s, x, p)=p+\frac{m \omega_{c}}{2}(\theta(s+\tau)-\theta(s))^{\perp} x .
$$

Notice that $\left\{x, p-\frac{m \omega_{c}}{2} \theta(s)^{\perp} x\right\}$ is a complete family of functional independent prime integrals of (22). We introduce the average operator along the characteristic flow (23) cf. [6]

$$
\begin{aligned}
\langle u\rangle(s, x, p) & =\frac{1}{T} \int_{0}^{T} u(S(\tau ; s, x, p), X(\tau ; s, x, p), P(\tau ; s, x, p)) \mathrm{d} \tau \\
& =\frac{1}{T} \int_{0}^{T} u\left(s+\tau, x, p+\frac{m \omega_{c}}{2}(\theta(s+\tau)-\theta(s))^{\perp} x\right) \mathrm{d} \tau \\
& =\frac{1}{T} \int_{0}^{T} u\left(\tau, x, p-\frac{m \omega_{c}}{2} \theta(s){ }^{\perp} x+\frac{m \omega_{c}}{2} \theta(\tau){ }^{\perp} x\right) \mathrm{d} \tau
\end{aligned}
$$

for any function $u \in L_{\#}^{2}\left(\mathbb{R}_{s} ; L^{2}\left(\mathbb{R}_{x}^{3} \times \mathbb{R}_{p}^{3}\right)\right)$.

Proposition 3.1 The average operator is linear continuous. It coincides with the orthogonal projection on the kernel of $\mathcal{T}$ i.e.,

$$
\langle u\rangle \in \operatorname{ker} \mathcal{T}: \int_{0}^{T} \int_{\mathbb{R}^{3}} \int_{\mathbb{R}^{3}}(u-\langle u\rangle) \varphi \mathrm{d} p \mathrm{~d} x \mathrm{~d} s=0, \quad \forall \varphi \in \operatorname{ker} \mathcal{T} .
$$

Proof. For any function $u \in L_{\#}^{2}\left(\mathbb{R}_{s} ; L^{2}\left(\mathbb{R}_{x}^{3} \times \mathbb{R}_{p}^{3}\right)\right)$ we have

$$
|\langle u\rangle|^{2}(s, x, p) \leq \frac{1}{T} \int_{0}^{T}|u|^{2}\left(s+\tau, x, p+\frac{m \omega_{c}}{2}(\theta(s+\tau)-\theta(s))^{\perp} x\right) \mathrm{d} \tau
$$

implying that

$$
\begin{aligned}
\int_{\mathbb{R}^{3}} \int_{\mathbb{R}^{3}}|\langle u\rangle|^{2}(s) \mathrm{d} p \mathrm{~d} x & \leq \frac{1}{T} \int_{0}^{T} \int_{\mathbb{R}^{3}} \int_{\mathbb{R}^{3}}|u|^{2}\left(s+\tau, x, p+\frac{m \omega_{c}}{2}(\theta(s+\tau)-\theta(s)){ }^{\perp} x\right) \mathrm{d} p \mathrm{~d} x \mathrm{~d} \tau \\
& =\frac{1}{T} \int_{0}^{T} \int_{\mathbb{R}^{3}} \int_{\mathbb{R}^{3}}|u|^{2}(s+\tau, x, p) \mathrm{d} p \mathrm{~d} x \mathrm{~d} \tau \\
& =\frac{1}{T}\|u\|^{2}
\end{aligned}
$$

Therefore we have

$$
\|\langle u\rangle\| \leq\|u\|, \quad \forall u \in L_{\#}^{2}\left(\mathbb{R}_{s} ; L^{2}\left(\mathbb{R}_{x}^{3} \times \mathbb{R}_{p}^{3}\right)\right)
$$

saying that $\langle\cdot\rangle \in \mathcal{L}\left(L_{\#}^{2}\left(\mathbb{R}_{s} ; L^{2}\left(\mathbb{R}_{x}^{3} \times \mathbb{R}_{p}^{3}\right)\right), L_{\#}^{2}\left(\mathbb{R}_{s} ; L^{2}\left(\mathbb{R}_{x}^{3} \times \mathbb{R}_{p}^{3}\right)\right)\right)$. It is well known that the kernel of $\mathcal{T}$ is given by the functions invariant along the characteristics (23) $\operatorname{ker} \mathcal{T}=\left\{u \in L_{\#}^{2}\left(\mathbb{R}_{s} ; L^{2}\left(\mathbb{R}_{x}^{3} \times \mathbb{R}_{p}^{3}\right)\right): \exists v\right.$ such that $\left.u(s, x, p)=v\left(x, p-\frac{m \omega_{c}}{2} \theta(s){ }^{\perp} x\right)\right\}$ 
Clearly $\langle u\rangle$ depends only on $x$ and $p-\frac{m \omega_{c}}{2} \theta(s)^{\perp} x$, cf. (24), and thus $\langle u\rangle$ belongs to $\operatorname{ker} \mathcal{T}$. Pick a function $\varphi \in \operatorname{ker} \mathcal{T}$ i.e.,

$$
\exists \psi: \varphi(s, x, p)=\psi\left(x, p-\frac{m \omega_{c}}{2} \theta(s)^{\perp} x\right)
$$

and let us compute $I=\int_{0}^{T} \int_{\mathbb{R}^{3}} \int_{\mathbb{R}^{3}}(u-\langle u\rangle) \varphi \mathrm{d} p \mathrm{~d} x \mathrm{~d} s$. Using the change of coordinates $q=p-\frac{m \omega_{c}}{2} \theta(s)^{\perp} x$, for fixed $(s, x)$, one gets

$$
\begin{aligned}
I & =\int_{0}^{T} \int_{\mathbb{R}^{3}} \int_{\mathbb{R}^{3}}(u-\langle u\rangle)\left(s, x, q+\frac{m \omega_{c}}{2} \theta(s){ }^{\perp} x\right) \psi(x, q) \mathrm{d} q \mathrm{~d} x \mathrm{~d} s \\
& =\int_{\mathbb{R}^{3}} \int_{\mathbb{R}^{3}} \psi(x, q)\left\{\int_{0}^{T} u\left(s, x, q+\frac{m \omega_{c}}{2} \theta(s)^{\perp} x\right) \mathrm{d} s-T\langle u\rangle\right\} \mathrm{d} q \mathrm{~d} x \\
& =0
\end{aligned}
$$

and therefore $\langle u\rangle=\operatorname{Proj}_{\text {ker } \mathcal{T}} u$ for any $u \in L_{\#}^{2}\left(\mathbb{R}_{s} ; L^{2}\left(\mathbb{R}_{x}^{3} \times \mathbb{R}_{p}^{3}\right)\right)$. In particular $\langle u\rangle=u$ for any $u \in \operatorname{ker} \mathcal{T}$.

We investigate now the solvability of the equation $\mathcal{T} u=v$. We have a simple characterization in terms of the kernel of the average operator. Notice that if $v=\mathcal{T} u \in$ Range $\mathcal{T}$ we have for any $\varphi \in \operatorname{ker} \mathcal{T}$

$$
\int_{0}^{T} \int_{\mathbb{R}^{3}} \int_{\mathbb{R}^{3}}(v-0) \varphi \mathrm{d} p \mathrm{~d} x \mathrm{~d} s=\int_{0}^{T} \int_{\mathbb{R}^{3}} \int_{\mathbb{R}^{3}} \mathcal{T} u \varphi \mathrm{d} p \mathrm{~d} x \mathrm{~d} s=-\int_{0}^{T} \int_{\mathbb{R}^{3}} \int_{\mathbb{R}^{3}} u \mathcal{T} \varphi \mathrm{d} p \mathrm{~d} x \mathrm{~d} s=0
$$

saying by Proposition 3.1 that $\langle v\rangle=0$. Moreover we have

Proposition 3.2 The restriction of $\mathcal{T}$ to $\operatorname{ker}\langle\cdot\rangle$ is one to one map onto $\operatorname{ker}\langle\cdot\rangle$. Its inverse belongs to $\mathcal{L}(\operatorname{ker}\langle\cdot\rangle, \operatorname{ker}\langle\cdot\rangle)$ and we have the Poincaré inequality

$$
\|u\| \leq T\|\mathcal{T} u\| \text { for any } u \in D(\mathcal{T}) \cap \operatorname{ker}\langle\cdot\rangle \text {. }
$$

Proof. We already know that Range $\mathcal{T} \subset \operatorname{ker}\langle\cdot\rangle$. Assume now that $u \in D(\mathcal{T}) \cap \operatorname{ker}\langle\cdot\rangle$ such that $\mathcal{T} u=0$. Since $\langle\cdot\rangle=\operatorname{Proj}_{\text {ker } \mathcal{T}}$ we have $u=\langle u\rangle=0$ saying that $\left.\mathcal{T}\right|_{\text {ker }\langle\cdot\rangle}$ is injective. Consider now $v \in \operatorname{ker}\langle\cdot\rangle$ and let us prove that there is $u \in \operatorname{ker}\langle\cdot\rangle \cap D(\mathcal{T})$ such that $\mathcal{T} u=v$. For any $\alpha>0$ there is a unique $u_{\alpha} \in D(\mathcal{T})$ such that

$$
\alpha u_{\alpha}+\mathcal{T} u_{\alpha}=v
$$

Indeed it is easily seen that the solutions $\left(u_{\alpha}\right)_{\alpha>0}$ are given by

$$
u_{\alpha}(s, x, p)=\int_{\mathbb{R}_{-}} e^{\alpha \tau} v\left(s+\tau, x, p-\frac{m \omega_{c}}{2} \theta(s){ }^{\perp} x+\frac{m \omega_{c}}{2} \theta(s+\tau){ }^{\perp} x\right) \mathrm{d} \tau .
$$


Applying the average operator to (26) yields $\left\langle u_{\alpha}\right\rangle=0$ for any $\alpha>0$. We introduce the function

$$
V(\tau ; s, x, p)=\int_{\tau}^{0} v\left(s+r, x, p-\frac{m \omega_{c}}{2} \theta(s){ }^{\perp} x+\frac{m \omega_{c}}{2} \theta(s+r){ }^{\perp} x\right) \mathrm{d} r .
$$

Notice that for any fixed $(s, x, p)$ the function $\tau \rightarrow V(\tau ; s, x, p)$ is $T$ periodic, because $\langle v\rangle=0$ and thus $\|V(\tau)\| \leq T\|v\|$ for any $\tau \in \mathbb{R}$. Integrating by parts we obtain

$$
u_{\alpha}(s, x, p)=-\int_{\mathbb{R}_{-}} e^{\alpha \tau} \partial_{\tau} V \mathrm{~d} \tau=\int_{\mathbb{R}_{-}} \alpha e^{\alpha \tau} V(\tau ; s, x, p) \mathrm{d} s
$$

implying that

$$
\left\|u_{\alpha}\right\| \leq \int_{\mathbb{R}_{-}} \alpha e^{\alpha \tau}\|V(\tau)\| \mathrm{d} \tau \leq T\|v\|
$$

Extracting a sequence $\left(\alpha_{n}\right)_{n}$ such that $\lim _{n \rightarrow+\infty} \alpha_{n}=0, \lim _{n \rightarrow+\infty} u_{\alpha_{n}}=u$ weakly in $L_{\#}^{2}\left(\mathbb{R}_{s} ; L^{2}\left(\mathbb{R}_{x}^{3} \times \mathbb{R}_{p}^{3}\right)\right)$ we deduce easily that

$$
u \in D(\mathcal{T}), \quad \mathcal{T} u=v, \quad\langle u\rangle=0, \quad\|u\| \leq T\|v\|
$$

saying that $\left(\left.\mathcal{T}\right|_{\operatorname{ker}\langle\cdot\rangle}\right)^{-1}$ is bounded linear operator and $\left\|\left(\left.\mathcal{T}\right|_{\operatorname{ker}\langle\cdot\rangle}\right)^{-1}\right\|_{\mathcal{L}(\operatorname{ker}\langle\cdot\rangle, \operatorname{ker}\langle\cdot\rangle)} \leq T$.

Remark 3.1 Notice that $\mathcal{T}^{-1}$ leaves invariant the set of zero average functions, with compact support. Indeed, if $v \in \operatorname{ker}\langle\cdot\rangle$ has compact support, let us say $\operatorname{supp} v \subset$ $\{(s, x, p):|x| \leq R,|p| \leq R\}$ for some $R>0$, it is easily seen that for any $\alpha>0$, the function $u_{\alpha}$ in (26) has compact support (uniformly with respect to $\alpha$ )

$$
\operatorname{supp} u_{\alpha} \subset\left\{(s, x, p):|x| \leq R, \quad|p| \leq\left(1+m\left|\omega_{c}\right|\|\theta\|_{L^{\infty}}\right) R\right\}
$$

and therefore the weak limit $u=\lim _{\alpha \backslash 0} u_{\alpha}$ has compact support.

Notice that we have the orthogonal decomposition of $L_{\#}^{2}\left(\mathbb{R}_{s} ; L^{2}\left(\mathbb{R}_{x}^{3} \times \mathbb{R}_{p}^{3}\right)\right)$ into invariant functions along the characteristics (22) and zero average functions $u=\langle u\rangle+(u-\langle u\rangle)$, since by Proposition 3.1 we have

$$
\int_{0}^{T} \int_{\mathbb{R}^{3}} \int_{\mathbb{R}^{3}}(u-\langle u\rangle)\langle u\rangle \mathrm{d} p \mathrm{~d} x \mathrm{~d} s=0 .
$$

We end this section with the following Sobolev inequality 
Proposition 3.3 There is a constant $C=C(T)$ such that for any function $u \in D(\mathcal{T})$ we have

$$
\|u\|_{L_{\#}^{\infty}\left(\mathbb{R}_{s} ; L^{2}\left(\mathbb{R}_{x}^{3} \times \mathbb{R}_{p}^{3}\right)\right)} \leq C(T)(\|u\|+\|\mathcal{T} u\|) .
$$

In particular for any function $u \in D(\mathcal{T}) \cap \operatorname{ker}\langle\cdot\rangle$ we have

$$
\|u\|_{L_{\#}^{\infty}\left(\mathbb{R}_{s} ; L^{2}\left(\mathbb{R}_{x}^{3} \times \mathbb{R}_{p}^{3}\right)\right)} \leq C(T)(1+T)\|\mathcal{T} u\|
$$

Proof. Without loss of generality we can assume that the function $u$ is smooth (the general case follows by standard density arguments). For any $s \in \mathbb{R}$ and $t \in[s-T, s]$ we have

$$
\frac{d}{d s}\left\{u\left(s, x, p-\frac{m \omega_{c}}{2}(\theta(t)-\theta(s))^{\perp} x\right)\right\}=(\mathcal{T} u)\left(s, x, p-\frac{m \omega_{c}}{2}(\theta(t)-\theta(s))^{\perp} x\right) .
$$

After integration one gets

$u\left(s, x, p-\frac{m \omega_{c}}{2}(\theta(t)-\theta(s))^{{ }} x\right)=u(t, x, p)+\int_{t}^{s} \mathcal{T} u\left(\tau, x, p-\frac{m \omega_{c}}{2}(\theta(t)-\theta(\tau)){ }^{\perp} x\right)$

implying that

$$
\|u(s, \cdot, \cdot)\|_{L^{2}\left(\mathbb{R}^{3} \times \mathbb{R}^{3}\right)}^{2} \leq 2\|u(t, \cdot, \cdot)\|_{L^{2}\left(\mathbb{R}^{3} \times \mathbb{R}^{3}\right)}^{2}+2 T\|\mathcal{T} u\|^{2}
$$

Averaging with respect to $t$ over the period $[s-T, s]$ yields

$$
\|u\|_{L_{\#}^{\infty}\left(\mathbb{R}_{s} ; L^{2}\left(\mathbb{R}_{x}^{3} \times \mathbb{R}_{p}^{3}\right)\right)}^{2} \leq \frac{2}{T}\|u\|^{2}+2 T\|\mathcal{T} u\|^{2}
$$

and the first statement follows with $C(T)=\max \{\sqrt{2 / T}, \sqrt{2 T}\}$. Moreover, if $u \in$ $D(\mathcal{T}) \cap \operatorname{ker}\langle\cdot\rangle$ we know by Proposition 3.2 that $\|u\| \leq T\|\mathcal{T} u\|$ and therefore

$$
\|u\|_{L_{\#}^{\infty}\left(\mathbb{R}_{s} ; L^{2}\left(\mathbb{R}_{x}^{3} \times \mathbb{R}_{p}^{3}\right)\right)} \leq C(T)\{T\|\mathcal{T} u\|+\|\mathcal{T} u\|)=C(T)(1+T)\|\mathcal{T} u\|
$$

\section{Commutation properties of the average with re- spect to derivations}

We have seen that $\mathcal{T}^{-1}$ restricted to zero average functions is linear and continuous. In view of further regularity that we need for the asymptotic analysis of (5) we investigate 
now the action of $\mathcal{T}^{-1}$ and $\langle\cdot\rangle$ over subspaces of smooth functions. We formulate our statements in the general framework of a characteristic flow associated to smooth, divergence free fields. Let $b: \mathbb{R}^{m} \rightarrow \mathbb{R}^{m}$ be a field satisfying

$$
b \in W_{\mathrm{loc}}^{1, \infty}\left(\mathbb{R}^{m}\right), \quad \operatorname{div}_{y} b=0
$$

and the growth condition

$$
\exists C>0:|b(y)| \leq C(1+|y|), \quad y \in \mathbb{R}^{m} .
$$

Under the above hypotheses the characteristic flow $Y=Y(s ; y)$ is well defined

$$
\begin{gathered}
\frac{d Y}{d s}=b(Y(s ; y)), \quad(s, y) \in \mathbb{R} \times \mathbb{R}^{m} \\
Y(0 ; y)=y, \quad y \in \mathbb{R}^{m},
\end{gathered}
$$

and has the regularity $Y \in W_{\text {loc }}^{1, \infty}\left(\mathbb{R} \times \mathbb{R}^{m}\right)$. Since the field is divergence free, we deduce by Liouville's theorem that for any $s \in \mathbb{R}$, the map $y \rightarrow Y(s ; y)$ is measure preserving. Notice that we don't make any periodicity assumption on the flow $Y$. As usual the notation $b \cdot \nabla_{y}$ stands for the first order differential operator with domain

$$
\mathrm{D}\left(b \cdot \nabla_{y}\right)=\left\{u \in L^{2}\left(\mathbb{R}^{m}\right): \operatorname{div}_{y}(u(y) b(y)) \in L^{2}\left(\mathbb{R}^{m}\right)\right\}
$$

which maps any $u \in \mathrm{D}\left(b \cdot \nabla_{y}\right)$ to the function $\operatorname{div}_{y}(u(y) b(y))$. The kernel of this operator is given by $L^{2}$ functions which are constant along the flow $Y$

$$
\operatorname{ker}\left(b \cdot \nabla_{y}\right)=\left\{u \in L^{2}\left(\mathbb{R}^{m}\right): u(Y(s ; y))=u(y), s \in \mathbb{R}, \text { a.e. } y \in \mathbb{R}^{m}\right\} .
$$

It is easily seen that for any function $u \in L^{2}\left(\mathbb{R}^{m}\right)$ and any $T>0$

$$
\left\|\frac{1}{T} \int_{0}^{T} u(Y(s ; \cdot)) \mathrm{d} s\right\|_{L^{2}\left(\mathbb{R}^{m}\right)} \leq\|u\|_{L^{2}\left(\mathbb{R}^{m}\right)}
$$

and therefore we can expect compactness properties for the family of averages along the flow $Y$ i.e., $\left\{\frac{1}{T} \int_{0}^{T} u(Y(s ; \cdot)) \mathrm{d} s, T>0\right\}$. Indeed, the mean ergodic theorem, or von Neumann's ergodic theorem (see [18], pp. 57) allows us to construct the average operator along the flow $Y$.

Proposition 4.1 For any function $u \in L^{2}\left(\mathbb{R}^{m}\right)$ the averages $\frac{1}{T} \int_{0}^{T} u(Y(s ; \cdot)) \mathrm{d} s$ converge strongly in $L^{2}\left(\mathbb{R}^{m}\right)$, when $T \rightarrow+\infty$, towards some function denoted $\langle u\rangle \in$ 
$L^{2}\left(\mathbb{R}^{m}\right)$. The map $\langle\cdot\rangle: L^{2}\left(\mathbb{R}^{m}\right) \rightarrow L^{2}\left(\mathbb{R}^{m}\right)$ is linear, continuous and coincides with the orthogonal projection on $\operatorname{ker}\left(b \cdot \nabla_{y}\right)$

$$
\langle u\rangle \in \operatorname{ker}\left(b \cdot \nabla_{y}\right): \int_{\mathbb{R}^{m}}(u(y)-\langle u\rangle(y)) \varphi(y) \mathrm{d} y=0, \forall \varphi \in \operatorname{ker}\left(b \cdot \nabla_{y}\right) .
$$

It is easily seen that Range $\left(b \cdot \nabla_{y}\right) \subset \operatorname{ker}\langle\cdot\rangle$. Actually it is shown in $[6]$ that $\overline{\operatorname{Range}\left(b \cdot \nabla_{y}\right)}=$ ker $\langle\cdot\rangle$. We are searching now for derivations commuting with the average operator. We prove that the average operator is commuting with any derivation $c \cdot \nabla_{y}$ associated to a field $c$ in involution with $b$. We recall here the following basic results concerning derivation operators along fields in $\mathbb{R}^{m}$. For any $\xi=\left(\xi_{1}(y), \ldots, \xi_{m}(y)\right)$, where $y \in \mathbb{R}^{m}$, we denote by $L_{\xi}$ the operator $\xi \cdot \nabla_{y}$. A direct computation shows that for any smooth fields $\xi, \eta$, the commutator between $L_{\xi}, L_{\eta}$ is still a first order operator, given by $\left[L_{\xi}, L_{\eta}\right]:=L_{\xi} L_{\eta}-L_{\eta} L_{\xi}=L_{\chi}$, where $\chi$ is the Poisson bracket of $\xi$ and $\eta$

$$
\chi=[\xi, \eta], \quad[\xi, \eta]_{i}=\left(\xi \cdot \nabla_{y}\right) \eta_{i}-\left(\eta \cdot \nabla_{y}\right) \xi_{i}=L_{\xi}\left(\eta_{i}\right)-L_{\eta}\left(\xi_{i}\right), \quad i \in\{1, \ldots, m\} .
$$

It is well known (see [2], pp. 93) that $L_{\xi}, L_{\eta}$ commute (or equivalently the Poisson bracket $[\xi, \eta]$ vanishes) iff the flows corresponding to $\xi, \eta$, let say $Z_{1}, Z_{2}$, commute

$$
Z_{1}\left(s_{1} ; Z_{2}\left(s_{2} ; y\right)\right)=Z_{2}\left(s_{2} ; Z_{1}\left(s_{1} ; y\right)\right), \quad s_{1}, s_{2} \in \mathbb{R}, \quad y \in \mathbb{R}^{m}
$$

Consider a smooth field $c$ in involution with $b$ and having bounded divergence

$$
c \in W_{\mathrm{loc}}^{1, \infty}\left(\mathbb{R}^{m}\right), \quad \operatorname{div}_{y} c \in L^{\infty}\left(\mathbb{R}^{m}\right), \quad[c, b]=0
$$

and let us denote by $Z$ the flow associated to $c$ (we assume that $Z$ is well defined for any $\left.(s, y) \in \mathbb{R} \times \mathbb{R}^{m}\right)$. We claim that the following commutation property holds true.

Proposition 4.2 Assume that $c$ is a smooth field in involution with b, with bounded divergence and well defined flow. Then the average operator commutes with the translations along the flow of $c$

$$
\langle u \circ Z(h ; \cdot)\rangle=\langle u\rangle \circ Z(h ; \cdot), \quad u \in L^{2}\left(\mathbb{R}^{m}\right), \quad h \in \mathbb{R} .
$$


Proof. The commutation property of the flows $Y, Z$ and Proposition 4.1 allow us to write the strong convergences in $L^{2}\left(\mathbb{R}^{m}\right)$

$$
\begin{aligned}
\langle u \circ Z(h ; \cdot)\rangle & =\lim _{T \rightarrow+\infty} \frac{1}{T} \int_{0}^{T} u \circ Z(h ; Y(s ; \cdot)) \mathrm{d} s \\
& =\lim _{T \rightarrow+\infty} \frac{1}{T} \int_{0}^{T} u \circ Y(s ; Z(h ; \cdot)) \mathrm{d} s \\
& =\left(\lim _{T \rightarrow+\infty} \frac{1}{T} \int_{0}^{T} u(Y(s ; \cdot)) \mathrm{d} s\right) \circ Z(h ; \cdot) \\
& =\langle u\rangle \circ Z(h ; \cdot) .
\end{aligned}
$$

Notice that the third equality in the above computations follows by changing the variable along the flow $Z$ and by using the boundedness of $\operatorname{div}_{y} c$.

We denote by $c \cdot \nabla_{y}$ the operator with the domain

$$
\mathrm{D}\left(c \cdot \nabla_{y}\right)=\left\{u \in L^{2}\left(\mathbb{R}^{m}\right): \operatorname{div}_{y}(u(y) c(y)) \in L^{2}\left(\mathbb{R}^{m}\right)\right\}
$$

which maps any function $u \in \mathrm{D}\left(c \cdot \nabla_{y}\right)$ to the function $\operatorname{div}_{y}(u c)-u \operatorname{div}_{y} c$.

Proposition 4.3 Under the hypotheses of Proposition 4.2, assume that $u \in \mathrm{D}\left(c \cdot \nabla_{y}\right)$. Then $\langle u\rangle \in \mathrm{D}\left(c \cdot \nabla_{y}\right)$ and $c \cdot \nabla_{y}\langle u\rangle=\left\langle c \cdot \nabla_{y} u\right\rangle$.

Proof. For any $h \in \mathbb{R}^{\star}$ we have

$$
\frac{\langle u\rangle \circ Z(h ; \cdot)-\langle u\rangle}{h}=\frac{\langle u \circ Z(h ; \cdot)\rangle-\langle u\rangle}{h}=\left\langle\frac{u \circ Z(h ; \cdot)-u}{h}\right\rangle .
$$

Since $u \in \mathrm{D}\left(c \cdot \nabla_{y}\right)$ we have the strong convergence $\lim _{h \rightarrow 0}(u \circ Z(h ; \cdot)-u) / h=c \cdot \nabla_{y} u$ in $L^{2}\left(\mathbb{R}^{m}\right)$ and by the continuity of the average operator, we deduce that

$$
\lim _{h \rightarrow 0} \frac{\langle u\rangle \circ Z(h ; \cdot)-\langle u\rangle}{h}=\left\langle c \cdot \nabla_{y} u\right\rangle
$$

strongly in $L^{2}\left(\mathbb{R}^{m}\right)$, saying that $\langle u\rangle \in \mathrm{D}\left(c \cdot \nabla_{y}\right)$ and $c \cdot \nabla_{y}\langle u\rangle=\left\langle c \cdot \nabla_{y} u\right\rangle$.

Similarly we can prove

Proposition 4.4 Assume that $u \in W^{1, p}\left([0, T] ; L^{2}\left(\mathbb{R}^{m}\right)\right)$ for some $p \in(1,+\infty)$. Then the application $(t, y) \rightarrow\langle u(t, \cdot)\rangle(y)$ belongs to $W^{1, p}\left([0, T] ; L^{2}\left(\mathbb{R}^{m}\right)\right)$ and we have $\partial_{t}\langle u\rangle=$ $\left\langle\partial_{t} u\right\rangle$. 
We are ready now to study how the regularity propagates under the action of $\left(b \cdot \nabla_{y}\right)^{-1}$. We make the following assumption (Poincaré inequality)

$$
\exists C_{P}>0 \text { such that }\|u\|_{L^{2}\left(\mathbb{R}^{m}\right)} \leq C_{P}\left\|b \cdot \nabla_{y} u\right\|_{L^{2}\left(\mathbb{R}^{m}\right)}, \forall u \in \mathrm{D}\left(b \cdot \nabla_{y}\right) \cap \operatorname{ker}\langle\cdot\rangle
$$

meaning that the range of $b \cdot \nabla_{y}$ is closed i.e., Range $\left(b \cdot \nabla_{y}\right)=\overline{\operatorname{Range}\left(b \cdot \nabla_{y}\right)}=\operatorname{ker}\langle\cdot\rangle$ and $b \cdot \nabla_{y}$ restricted to $\operatorname{ker}\langle\cdot\rangle$ is one to one map onto $\operatorname{ker}\langle\cdot\rangle$ with bounded inverse. Notice that the above hypothesis is satisfied by the operator in (21), cf. Proposition 3.2.

Proposition 4.5 Under the hypotheses of Proposition 4.2 and (30) assume that $v \in$ $\operatorname{ker}\langle\cdot\rangle \cap \mathrm{D}\left(c \cdot \nabla_{y}\right)$. Let us denote by $u$ the unique zero average solution of $b \cdot \nabla_{y} u=v$. Then $u \in \mathrm{D}\left(c \cdot \nabla_{y}\right)$ and $\left\|c \cdot \nabla_{y} u\right\|_{L^{2}\left(\mathbb{R}^{m}\right)} \leq C_{P}\left\|c \cdot \nabla_{y} v\right\|_{L^{2}\left(\mathbb{R}^{m}\right)}$.

Proof. For any $h \in \mathbb{R}^{\star}$ we have $u_{h}:=u \circ Z(h ; \cdot) \in \mathrm{D}\left(b \cdot \nabla_{y}\right)$ and $b \cdot \nabla_{y} u_{h}=\left(b \cdot \nabla_{y} u\right)_{h}=$ $v_{h}$. Therefore we deduce that $b \cdot \nabla_{y}\left(u_{h}-u\right)=v_{h}-v$. Since the average is commuting with the translations along the flow of $c$ we have

$$
\left\langle u_{h}-u\right\rangle=\left\langle u_{h}\right\rangle-\langle u\rangle=\langle u\rangle_{h}-\langle u\rangle=0-0=0
$$

and we can apply the Poincaré inequality (30)

$$
\left\|u_{h}-u\right\|_{L^{2}\left(\mathbb{R}^{m}\right)} \leq C_{P}\left\|v_{h}-v\right\|_{L^{2}\left(\mathbb{R}^{m}\right)}, h \in \mathbb{R}^{\star}
$$

Therefore $\sup _{h \in \mathbb{R}^{*}}\left\|u_{h}-u\right\|_{L^{2}\left(\mathbb{R}^{m}\right)} /|h| \leq C_{P}\left\|c \cdot \nabla_{y} v\right\|_{L^{2}\left(\mathbb{R}^{m}\right)}$ saying that $u \in \mathrm{D}\left(c \cdot \nabla_{y}\right)$ and $\left\|c \cdot \nabla_{y} u\right\|_{L^{2}\left(\mathbb{R}^{m}\right)} \leq C_{P}\left\|c \cdot \nabla_{y} v\right\|_{L^{2}\left(\mathbb{R}^{m}\right)}$. Notice also that we have

$$
b \cdot \nabla_{y}\left(c \cdot \nabla_{y} u\right)=c \cdot \nabla_{y}\left(b \cdot \nabla_{y} u\right)=c \cdot \nabla_{y} v
$$

with $\left\langle c \cdot \nabla_{y} u\right\rangle=c \cdot \nabla_{y}\langle u\rangle=0$ saying that $\left(b \cdot \nabla_{y}\right)^{-1}\left(c \cdot \nabla_{y} v\right)=c \cdot \nabla_{y} u$.

\subsection{Regularity propagation under fast oscillating magnetic fields}

We apply the previous general results to the operator (21) acting on the phase space $(s, x, p) \in \mathbb{R}^{7}$. We indicate a complete family of fields in involution with respect to $\partial_{s}+\frac{m \omega_{c}}{2} \theta^{\prime}(s)^{\perp} x \cdot \nabla_{p}$. The reader can convince himself by direct computations. 
Proposition 4.6 The following fields are in involution with respect to $\partial_{s}+\frac{m \omega_{c}}{2} \theta^{\prime}(s){ }^{\perp} x$. $\nabla_{p}$

$$
\begin{gathered}
c^{1} \cdot \nabla_{(s, x, p)}=\partial_{x_{1}}-\frac{m \omega_{c}}{2} \theta(s) \partial_{p_{2}}, \quad c^{2} \cdot \nabla_{(s, x, p)}=\partial_{x_{2}}+\frac{m \omega_{c}}{2} \theta(s) \partial_{p_{1}}, \quad c^{3} \cdot \nabla_{(s, x, p)}=\partial_{x_{3}} \\
c^{4} \cdot \nabla_{(s, x, p)}=\partial_{p_{1}}, \quad c^{5} \cdot \nabla_{(s, x, p)}=\partial_{p_{2}}, \quad c^{6} \cdot \nabla_{(s, x, p)}=\partial_{p_{3}} .
\end{gathered}
$$

Proposition 4.7 Assume that $v \in \operatorname{ker}\langle\cdot\rangle$ such that $\nabla_{x} v, \nabla_{p} v \in\left(L_{\#}^{2}\left(\mathbb{R}_{s} ; L^{2}\left(\mathbb{R}_{x}^{3} \times\right.\right.\right.$ $\left.\left.\mathbb{R}_{p}^{3}\right)\right)^{3}$. Let us denote by $u$ the unique zero average solution of $\mathcal{T} u=v$. Then $\nabla_{x} u, \nabla_{p} u \in\left(L_{\#}^{2}\left(\mathbb{R}_{s} ; L^{2}\left(\mathbb{R}_{x}^{3} \times \mathbb{R}_{p}^{3}\right)\right)\right)^{3}$ and

$$
\begin{gathered}
\left\|\partial_{x_{1}} u\right\| \leq T\left\{\left\|\partial_{x_{1}} v\right\|+m\left|\omega_{c}\right|\|\theta\|_{L^{\infty}\left(\mathbb{R}^{3}\right)}\left\|\partial_{p_{2}} v\right\|\right\} \\
\left\|\partial_{x_{2}} u\right\| \leq T\left\{\left\|\partial_{x_{2}} v\right\|+m\left|\omega_{c}\right|\|\theta\|_{L^{\infty}\left(\mathbb{R}^{3}\right)}\left\|\partial_{p_{1}} v\right\|\right\} \\
\left\|\partial_{x_{3}} u\right\| \leq T\left\|\partial_{x_{3}} v\right\|, \quad\left\|\partial_{p_{i}} u\right\| \leq T\left\|\partial_{p_{i}} v\right\|, \quad i \in\{1,2,3\} .
\end{gathered}
$$

Proof. By the hypotheses we know that $v \in \cap_{i=1}^{6} \mathrm{D}\left(c^{i} \cdot \nabla_{(s, x, p)}\right)$ and therefore Proposition 4.5 implies

$$
u \in \cap_{i=1}^{6} \mathrm{D}\left(c^{i} \cdot \nabla_{(s, x, p)}\right), \quad\left\|c^{i} \cdot \nabla_{(s, x, p)} u\right\| \leq T\left\|c^{i} \cdot \nabla_{(s, x, p)} v\right\|, \quad i \in\{1, \ldots, 6\}
$$

In particular we have $\nabla_{x} u, \nabla_{p} u \in\left(L_{\#}^{2}\left(\mathbb{R}_{s} ; L^{2}\left(\mathbb{R}_{x}^{3} \times \mathbb{R}_{p}^{3}\right)\right)\right)^{3}$

$$
\begin{aligned}
\left\|\partial_{x_{3}} u\right\| & \leq T\left\|\partial_{x_{3}} v\right\|, \quad\left\|\partial_{p_{i}} u\right\| \leq T\left\|\partial_{p_{i}} v\right\|, \quad i \in\{1,2,3\} \\
\left\|\partial_{x_{1}} u\right\| & =\left\|c^{1} \cdot \nabla_{(s, x, p)} u+\frac{m \omega_{c}}{2} \theta \partial_{p_{2}} u\right\| \\
& \leq\left\|c^{1} \cdot \nabla_{(s, x, p)} u\right\|+\frac{m\left|\omega_{c}\right|}{2}\|\theta\|_{L^{\infty}\left(\mathbb{R}^{3}\right)}\left\|\partial_{p_{2}} u\right\| \\
& \leq T\left\|c^{1} \cdot \nabla_{(s, x, p)} v\right\|+T \frac{m\left|\omega_{c}\right|}{2}\|\theta\|_{L^{\infty}\left(\mathbb{R}^{3}\right)}\left\|\partial_{p_{2}} v\right\| \\
& \leq T\left\{\left\|\partial_{x_{1}} v\right\|+m\left|\omega_{c}\right|\|\theta\|_{L^{\infty}\left(\mathbb{R}^{3}\right)}\left\|\partial_{p_{2}} v\right\|\right\}
\end{aligned}
$$

and similarly $\left\|\partial_{x_{2}} u\right\| \leq T\left\{\left\|\partial_{x_{2}} v\right\|+m\left|\omega_{c}\right|\|\theta\|_{L^{\infty}\left(\mathbb{R}^{3}\right)}\left\|\partial_{p_{1}} v\right\|\right\}$. 


\section{Limit model}

We are ready now to investigate the limit model of (5) as $\varepsilon \searrow 0$. We appeal to the method introduced in [6] for general transport problems (see also [4]) which combines Hilbert expansion and average properties. A rigorous convergence result is obtained using the notion of two-scale convergence. We analyze the properties of the limit model, in particular we establish the energy conservation and justify the confinement around the magnetic lines. The terms in the Hilbert expansion (17) satisfy

$$
\begin{gathered}
\mathcal{T} f^{0}=0 \\
\partial_{t} f^{0}+\frac{p}{m} \cdot \nabla_{x} f^{0}+\left(e E(t, x)+\omega_{c} \theta(s){ }^{\perp} p\right) \cdot \nabla_{p} f^{0}+\mathcal{T} f^{1}=0 .
\end{gathered}
$$

The equation (31) says that at any time $t \in \mathbb{R}_{+}$the function $(s, x, p) \rightarrow f^{0}(t, s, x, p)$ belongs to $\operatorname{ker} \mathcal{T}$ and therefore

$$
f^{0}(t, s, x, p)=g^{0}\left(t, x, p-\frac{m \omega_{c}}{2} \theta(s)^{\perp} x\right)
$$

The time evolution equation for $f^{0}$ is obtained from (32) after eliminating $f^{1}$. Thanks to Proposition 3.2 we have for any $t \in \mathbb{R}_{+}$

$$
\partial_{t} f^{0}+\frac{p}{m} \cdot \nabla_{x} f^{0}+\left(e E(t, x)+\omega_{c} \theta(s){ }^{\perp} p\right) \cdot \nabla_{p} f^{0} \in \text { Range } \mathcal{T}=\operatorname{ker}\langle\cdot\rangle .
$$

Therefore (32) is equivalent to

$$
\left\langle\partial_{t} f^{0}+\frac{p}{m} \cdot \nabla_{x} f^{0}+\left(e E(t, x)+\omega_{c} \theta(s){ }^{\perp} p\right) \cdot \nabla_{p} f^{0}\right\rangle=0, \quad t \in \mathbb{R}_{+} .
$$

It is easily seen that $\left\langle\partial_{t} f^{0}\right\rangle=\partial_{t}\left\langle f^{0}\right\rangle=\partial_{t} f^{0}$. It remains to compute the averages of the derivatives with respect to $x$ and $p$. For simplifying the computations we assume that $f^{0}$ is smooth but it can be shown that the limit model which we will obtain still holds true in the distribution sense.

Lemma 5.1 Assume that $f(s, x, p)=g\left(x, q=p-\frac{m \omega_{c}}{2} \theta(s)^{\perp} x\right)$ is smooth. Then we have

$$
\begin{aligned}
& \left\langle\frac{p}{m} \cdot \nabla_{x} f\right\rangle=\left(\frac{q}{m}+\frac{\omega_{c}}{2}\langle\theta\rangle{ }^{\perp} x\right) \cdot \nabla_{x} g+\left(\frac{\omega_{c}}{2}\langle\theta\rangle q+\frac{m \omega_{c}^{2}}{4}\left\langle\theta^{2}\right\rangle{ }^{\perp} x\right) \cdot{ }^{\perp} \nabla_{q} g \\
= & \left(\frac{p}{m}-\frac{\omega_{c}}{2}(\theta-\langle\theta\rangle)^{\perp} x\right) \cdot \nabla_{x} f+\left(\frac{\omega_{c}}{2}(\theta-\langle\theta\rangle){ }^{\perp} p+\frac{m \omega_{c}^{2}}{4}\left(2 \theta\langle\theta\rangle-\left\langle\theta^{2}\right\rangle-\theta^{2}\right)^{\perp \perp} x\right) \cdot \nabla_{p} f
\end{aligned}
$$


and

$$
\begin{aligned}
\left\langle\left(e E(x)+\omega_{c} \theta^{\perp} p\right) \cdot \nabla_{p} f\right\rangle & =e E \cdot \nabla_{q} g+\left(\omega_{c}\langle\theta\rangle{ }^{\perp} q+\frac{m \omega_{c}^{2}}{2}\left\langle\theta^{2}\right\rangle{ }^{\perp \perp} x\right) \cdot \nabla_{q} g \\
& =\left(e E+\omega_{c}\left\langle\theta{ }^{\perp} p+\frac{m \omega_{c}^{2}}{2}\left(\left\langle\theta^{2}\right\rangle-\theta\langle\theta\rangle\right)^{\perp \perp} x\right) \cdot \nabla_{p} f .\right.
\end{aligned}
$$

Proof. We have, with the notation ${ }^{\perp} \nabla_{q}=\left(\partial_{q_{2}},-\partial_{q_{1}}, 0\right)$

$$
\nabla_{x} f=\nabla_{x} g+\frac{m \omega_{c}}{2} \theta(s)^{\perp} \nabla_{q} g
$$

and therefore

$$
\left\langle\frac{p}{m} \cdot \nabla_{x} f\right\rangle=\langle p\rangle \cdot \frac{\nabla_{x} g}{m}+\frac{\omega_{c}}{2}\langle p \theta\rangle \cdot{ }^{\perp} \nabla_{q} g
$$

since the derivatives of $g$ are constant along the characteristic flow (22). By the definition of the average operator we have

$$
\begin{aligned}
\langle p\rangle & =\frac{1}{T} \int_{0}^{T}\left\{p-\frac{m \omega_{c}}{2} \theta(s)^{\perp} x+\frac{m \omega_{c}}{2} \theta(\tau){ }^{\perp} x\right\} \mathrm{d} \tau \\
& =q+\frac{m \omega_{c}}{2}\langle\theta\rangle^{\perp} x
\end{aligned}
$$

where $q=p-\frac{m \omega_{c}}{2} \theta(s)^{\perp} x$. Similarly we obtain

$$
\begin{aligned}
\langle p \theta\rangle & =\frac{1}{T} \int_{0}^{T}\left\{p-\frac{m \omega_{c}}{2} \theta(s)^{\perp} x+\frac{m \omega_{c}}{2} \theta(\tau){ }^{\perp} x\right\} \theta(\tau) \mathrm{d} \tau \\
& =q\langle\theta\rangle+\frac{m \omega_{c}}{2}\left\langle\theta^{2}\right\rangle{ }^{\perp} x
\end{aligned}
$$

and finally

$$
\left\langle\frac{p}{m} \cdot \nabla_{x} f\right\rangle=\left(\frac{q}{m}+\frac{\omega_{c}}{2}\langle\theta\rangle{ }^{\perp} x\right) \cdot \nabla_{x} g+\left(\frac{\omega_{c}}{2}\langle\theta\rangle q+\frac{m \omega_{c}^{2}}{4}\left\langle\theta^{2}\right\rangle{ }^{\perp} x\right) \cdot{ }^{\perp} \nabla_{q} g .
$$

The second statement follows easily observing that $\nabla_{p} f=\nabla_{q} g$ and therefore

$$
\begin{aligned}
\left\langle\left(e E(x)+\omega_{c} \theta^{\perp} p\right) \cdot \nabla_{p} f\right\rangle & =e E(x) \cdot \nabla_{q} g+\omega_{c}\left\langle\theta^{\perp} p\right\rangle \cdot \nabla_{q} g \\
& =e E(x) \cdot \nabla_{q} g+\left(\omega_{c}\langle\theta\rangle{ }^{\perp} q+\frac{m \omega_{c}^{2}}{2}\left\langle\theta^{2}\right\rangle{ }^{\perp \perp} x\right) \cdot \nabla_{q} g .
\end{aligned}
$$

Combining the previous computations yields the transport equation

$$
\partial_{t} g^{0}+\left(\frac{q}{m}+\frac{\omega_{c}}{2}\left\langle\theta{ }^{\perp} x\right) \cdot \nabla_{x} g^{0}+\left(e E+\frac{\omega_{c}}{2}\langle\theta\rangle^{\perp} q+\frac{m \omega_{c}^{2}}{4}\left\langle\theta^{2}\right\rangle{ }^{\perp \perp} x\right) \cdot \nabla_{q} g^{0}=0\right.
$$


which is exactly (12). Performing the change of unknown $f^{0}(t, s, x, p)=g^{0}(t, x, p-$ $\left.\frac{m \omega_{c}}{2} \theta(s)^{\perp} x\right)$ leads to

$$
\begin{gathered}
\partial_{t} f^{0}+\left(\frac{p}{m}-\frac{\omega_{c}}{2}(\theta(s)-\langle\theta\rangle)^{\perp} x\right) \cdot \nabla_{x} f^{0} \\
+\quad\left(e E(t, x)+\frac{\omega_{c}}{2}(\theta(s)+\langle\theta\rangle)^{\perp} p+\frac{m \omega_{c}^{2}}{4}\left(\left\langle\theta^{2}\right\rangle-\theta^{2}(s)\right)^{\perp \perp} x\right) \cdot \nabla_{p} f^{0}=0 .
\end{gathered}
$$

Based on the concept of two-scale convergence, introduced in [17] and developed in [1] we prove a two-scale convergence result of the solutions in (5) towards (35), supplemented by an appropriate initial condition.

Definition 5.1 Let $\left(f^{n}(t, x, p)\right)_{n \in \mathbb{N}^{\star}}$ be a sequence in $L^{2}\left([0, I] ; L^{2}\left(\mathbb{R}^{3} \times \mathbb{R}^{3}\right)\right)$. We say that $\left(f^{n}\right)_{n}$ two-scale converges towards some function $f^{0} \in L^{2}\left([0, I] ; L_{\#}^{2}\left(\mathbb{R}_{s} ; L^{2}\left(\mathbb{R}_{x}^{3} \times\right.\right.\right.$ $\left.\left.\mathbb{R}_{p}^{3}\right)\right)$ ) iff we have

$\lim _{n \rightarrow+\infty} \int_{0}^{I} \int_{\mathbb{R}^{3}} \int_{\mathbb{R}^{3}} f^{n}(t, x, p) \varphi(t, t / \varepsilon, x, p) \mathrm{d} p \mathrm{~d} x \mathrm{~d} t=\int_{0}^{I} \frac{1}{T} \int_{0}^{T} \int_{\mathbb{R}^{3}} \int_{\mathbb{R}^{3}}\left(f^{0} \varphi\right)(t, s, x, p) \mathrm{d} p \mathrm{~d} x \mathrm{~d} s \mathrm{~d} t$ for any test function $\varphi \in L^{2}\left([0, I] ; C_{\#}^{0}\left(\mathbb{R}_{s} ; L^{2}\left(\mathbb{R}^{3} \times \mathbb{R}^{3}\right)\right)\right.$ ) (here $C_{\#}^{0}\left(\mathbb{R}_{s} ; X\right)$ stands for the set of continuous $T$ periodic functions with values in the normed linear space $X)$.

Adapting the arguments in [1] we obtain

Proposition 5.1 Let $\left(f^{\varepsilon}(t, x, p)\right)_{\varepsilon>0}$ be a bounded family in $L^{2}\left([0, I] ; L^{2}\left(\mathbb{R}^{3} \times \mathbb{R}^{3}\right)\right)$. Then there is a sequence $\varepsilon_{n} \searrow 0$ and a function $f^{0} \in L^{2}\left([0, I] ; L_{\#}^{2}\left(\mathbb{R}_{s} ; L^{2}\left(\mathbb{R}_{x}^{3} \times \mathbb{R}_{p}^{3}\right)\right)\right)$ such that $\left(f^{\varepsilon_{n}}\right)_{n}$ two-scale converges towards $f^{0}$.

Proof. (of Theorem 2.1) We use the weak formulation of (5) with the test function $\eta(t) \varphi(t / \varepsilon, x, p)$ where $\eta \in C_{c}^{1}\left(\mathbb{R}_{+}\right)$and $\varphi \in C_{c}^{1}\left(\mathbb{R} / T \mathbb{Z} \times \mathbb{R}^{3} \times \mathbb{R}^{3}\right)$. We obtain

$$
\begin{aligned}
& -\eta(0) \int_{\mathbb{R}^{3}} \int_{\mathbb{R}^{3}} f^{\mathrm{in}}(x, p) \varphi(0, x, p) \mathrm{d} p \mathrm{~d} x-\int_{\mathbb{R}_{+}} \eta^{\prime}(t) \int_{\mathbb{R}^{3}} \int_{\mathbb{R}^{3}} f^{\varepsilon}(t, x, p) \varphi(t / \varepsilon, x, p) \mathrm{d} p \mathrm{~d} x \mathrm{~d} t \\
& -\int_{\mathbb{R}_{+}} \eta \int_{\mathbb{R}^{3}} \int_{\mathbb{R}^{3}} f^{\varepsilon}(t, x, p)\left[\frac{1}{\varepsilon} \partial_{s} \varphi(t / \varepsilon, x, p)+\frac{p}{m} \cdot \nabla_{x} \varphi(t / \varepsilon, x, p)\right] \mathrm{d} p \mathrm{~d} x \mathrm{~d} t \\
& -\int_{\mathbb{R}_{+}} \eta \int_{\mathbb{R}^{3}} \int_{\mathbb{R}^{3}} f^{\varepsilon}(t, x, p)\left[e E+\frac{m \omega_{c}}{2 \varepsilon} \theta^{\prime}(t / \varepsilon)^{\perp} x+\omega_{c} \theta(t / \varepsilon){ }^{\perp} p\right] \cdot \nabla_{p} \varphi(t / \varepsilon, x, p) \mathrm{d} p \mathrm{~d} x \mathrm{~d} t \\
& =0 .
\end{aligned}
$$

Multiplying the previous formulation by $\varepsilon$, one gets by two-scale convergence (after extraction eventually)

$$
\int_{\mathbb{R}_{+}} \eta(t) \frac{1}{T} \int_{0}^{T} \int_{\mathbb{R}^{3}} \int_{\mathbb{R}^{3}} f^{0}(t, s, x, p) \mathcal{T} \varphi(s, x, p) \mathrm{d} p \mathrm{~d} x \mathrm{~d} s \mathrm{~d} t=0
$$


saying that $f^{0}(t, \cdot, \cdot, \cdot) \in \operatorname{ker} \mathcal{T}$ for any $t \in \mathbb{R}_{+}$. We use now the same weak formulation, but with $\varphi \in C_{c}^{1}\left(\mathbb{R} / T \mathbb{Z} \times \mathbb{R}^{3} \times \mathbb{R}^{3}\right) \cap \operatorname{ker} \mathcal{T}$. For example take $\varphi(s, x, p)=\psi(x, p-$ $\left.\frac{m \omega_{c}}{2} \theta(s){ }^{\perp} x\right)$ with $\psi \in C_{c}^{1}\left(\mathbb{R}^{3} \times \mathbb{R}^{3}\right)$, let us say $\operatorname{supp} \psi \subset\{(x, q): \max \{|x|,|q|\} \leq R\}$. In this case $\operatorname{supp} \varphi \subset\left\{(s, x, p): \max \{|x|,|p|\} \leq\left(1+m\left|\omega_{c}\right|\|\theta\|_{L^{\infty}\left(\mathbb{R}^{3}\right)} / 2\right) R\right\}$. Since $\varphi(s, x, p) \in \operatorname{ker} \mathcal{T}$ we have

$$
\frac{1}{\varepsilon} \partial_{s} \varphi(t / \varepsilon, x, p)+\frac{m \omega_{c}}{2 \varepsilon} \theta^{\prime}(t / \varepsilon)^{\perp} x \cdot \nabla_{p} \varphi(t / \varepsilon, x, p)=0
$$

and the weak formulation becomes

$$
\begin{aligned}
& -\eta(0) \int_{\mathbb{R}^{3}} \int_{\mathbb{R}^{3}} f^{\text {in }}(x, p) \varphi(0, x, p) \mathrm{d} p \mathrm{~d} x-\int_{\mathbb{R}_{+}} \eta^{\prime}(t) \int_{\mathbb{R}^{3}} \int_{\mathbb{R}^{3}} f^{\varepsilon}(t, x, p) \varphi(t / \varepsilon, x, p) \mathrm{d} p \mathrm{~d} x \mathrm{~d} t \\
& -\int_{\mathbb{R}_{+}} \eta(t) \int_{\mathbb{R}^{3}} \int_{\mathbb{R}^{3}} f^{\varepsilon}\left[\frac{p}{m} \cdot \nabla_{x} \varphi(t / \varepsilon, x, p)+\left(e E+\omega_{c} \theta(t / \varepsilon)^{\perp} p\right) \cdot \nabla_{p} \varphi(t / \varepsilon, x, p)\right] \mathrm{d} p \mathrm{~d} x \mathrm{~d} t \\
& =0 .
\end{aligned}
$$

As before, by two-scale convergence one gets

$$
\begin{aligned}
& \lim _{\varepsilon \searrow 0} \int_{\mathbb{R}_{+}} \eta^{\prime}(t) \int_{\mathbb{R}^{3}} \int_{\mathbb{R}^{3}} f^{\varepsilon}(t, x, p) \varphi(t / \varepsilon, x, p) \mathrm{d} p \mathrm{~d} x \mathrm{~d} t \\
& =\int_{\mathbb{R}_{+}} \eta^{\prime}(t) \frac{1}{T} \int_{0}^{T} \int_{\mathbb{R}^{3}} \int_{\mathbb{R}^{3}} f^{0}(t, s, x, p) \varphi(s, x, p) \mathrm{d} p \mathrm{~d} x \mathrm{~d} s \mathrm{~d} t .
\end{aligned}
$$

Similarly one gets

$$
\begin{aligned}
& \lim _{\varepsilon \backslash 0} \int_{\mathbb{R}_{+}} \eta \int_{\mathbb{R}^{3}} \int_{\mathbb{R}^{3}} f^{\varepsilon}\left[\frac{p}{m} \cdot \nabla_{x} \varphi(t / \varepsilon, x, p)+\left(e E+\omega_{c} \theta(t / \varepsilon){ }^{\perp} p\right) \cdot \nabla_{p} \varphi(t / \varepsilon, x, p)\right] \mathrm{d} p \mathrm{~d} x \mathrm{~d} t \\
& =\int_{\mathbb{R}_{+}} \frac{\eta}{T} \int_{0}^{T} \int_{\mathbb{R}^{3}} \int_{\mathbb{R}^{3}} f^{0}(t, s, x, p)\left[\frac{p}{m} \cdot \nabla_{x} \varphi+\left(e E+\omega_{c} \theta(s){ }^{\perp} p\right) \cdot \nabla_{p} \varphi\right] \mathrm{d} p \mathrm{~d} x \mathrm{~d} s \mathrm{~d} t .
\end{aligned}
$$

Therefore (36) implies

$$
\begin{aligned}
& -\eta(0) \int_{\mathbb{R}^{3}} \int_{\mathbb{R}^{3}} f^{\text {in }}(x, p) \varphi(0, x, p) \mathrm{d} p \mathrm{~d} x-\int_{\mathbb{R}_{+}} \frac{\eta^{\prime}(t)}{T} \int_{0}^{T} \int_{\mathbb{R}^{3}} \int_{\mathbb{R}^{3}} f^{0}(t, s, x, p) \varphi(s, x, p) \mathrm{d} p \mathrm{~d} x \mathrm{~d} s \mathrm{~d} t \\
= & \int_{\mathbb{R}_{+}} \frac{\eta(t)}{T} \int_{0}^{T} \int_{\mathbb{R}^{3}} \int_{\mathbb{R}^{3}} f^{0}(t, s, x, p)\left[\frac{p}{m} \cdot \nabla_{x} \varphi+\left(e E+\omega_{c} \theta(s)^{\perp} p\right) \cdot \nabla_{p} \varphi\right] \mathrm{d} p \mathrm{~d} x \mathrm{~d} s \mathrm{~d} t .
\end{aligned}
$$

We transform the term involving the initial condition. Since $\varphi \in \operatorname{ker} \mathcal{T}$ we have for any $s \in \mathbb{R}$

$$
\varphi(0, x, p)=\varphi\left(s, x, p-\frac{m \omega_{c}}{2} \theta(0)^{\perp} x+\frac{m \omega_{c}}{2} \theta(s)^{\perp} x\right)
$$


implying that

$$
\begin{aligned}
T \int_{\mathbb{R}^{3}} \int_{\mathbb{R}^{3}} f^{\mathrm{in}} \varphi(0, x, p) \mathrm{d} p \mathrm{~d} x & =\int_{0}^{T} \int_{\mathbb{R}^{3}} \int_{\mathbb{R}^{3}} f^{\mathrm{in}}(x, p) \varphi\left(s, x, p-\frac{m \omega_{c}}{2}(\theta(0)-\theta(s))^{\perp} x\right) \mathrm{d} p \mathrm{~d} x \mathrm{~d} s \\
& =\int_{0}^{T} \int_{\mathbb{R}^{3}} \int_{\mathbb{R}^{3}} f^{\text {in }}\left(x, p-\frac{m \omega_{c}}{2}(\theta(s)-\theta(0))^{\perp} x\right) \varphi(s, x, p) \mathrm{d} p \mathrm{~d} x \mathrm{~d} s .
\end{aligned}
$$

We transform now the right hand side of (37) thanks to Lemma 5.1. Indeed, since $f(t, \cdot, \cdot, \cdot) \in \operatorname{ker} \mathcal{T}$, for any $t \in \mathbb{R}_{+}$, we have by the variational formulation of the average operator in Proposition 3.1

$$
\begin{aligned}
& \int_{0}^{T} \int_{\mathbb{R}^{3}} \int_{\mathbb{R}^{3}} f^{0}(t)\left[\frac{p}{m} \cdot \nabla_{x} \varphi+\left(e E+\omega_{c} \theta(s)^{\perp} p\right) \cdot \nabla_{p} \varphi\right] \mathrm{d} p \mathrm{~d} x \mathrm{~d} s \\
= & \int_{0}^{T} \int_{\mathbb{R}^{3}} \int_{\mathbb{R}^{3}} f^{0}\left\langle\frac{p}{m} \cdot \nabla_{x} \varphi+\left(e E+\omega_{c} \theta(s)^{\perp} p\right) \cdot \nabla_{p} \varphi\right\rangle \mathrm{d} p \mathrm{~d} x \mathrm{~d} s \\
= & \int_{0}^{T} \int_{\mathbb{R}^{3}} \int_{\mathbb{R}^{3}} f^{0}(t)\left[\left(\frac{p}{m}-\frac{\omega_{c}}{2}(\theta(s)-\langle\theta\rangle)^{\perp} x\right) \cdot \nabla_{x} \varphi\right. \\
+ & \left.\left(e E+\frac{\omega_{c}}{2}(\theta(s)+\langle\theta\rangle)^{\perp} p+\frac{m \omega_{c}^{2}}{4}\left(\left\langle\theta^{2}\right\rangle-\theta^{2}(s)\right)^{\perp \perp} x\right) \cdot \nabla_{p} \varphi\right] \mathrm{d} p \mathrm{~d} x \mathrm{~d} s .
\end{aligned}
$$

Combining now (37), (38), (39) implies that $f^{0}$ is a weak solution of the transport problem (13), (14). Equivalently, the function $g^{0}$ such that $f^{0}(t, s, x, p)=g^{0}(t, x, p-$ $\left.m \omega_{c} / 2 \theta(s)^{\perp} x\right)$ is a weak solution of the transport equation (34), supplemented by the initial condition

$$
g^{0}(0, x, q)=f^{\text {in }}\left(x, q+\frac{m \omega_{c}}{2} \theta(0)^{\perp} x\right)
$$

Notice that the model (34), (40) is posed in a six dimensional phase space whereas the model (13), (14) acts on a seven dimensional phase space. Thus, at least for the numerical point of view it is preferable to appeal to (34), (40).

It is interesting to observe that, as $\varepsilon \searrow 0$, the kinetic energy of $f^{0}(t, t / \varepsilon, \cdot, \cdot) \approx$ $f^{\varepsilon}(t, \cdot, \cdot)$ can be decomposed into kinetic energy and elastic energy associated to the density $g^{0}$

$$
W^{0}(t)=\int_{\mathbb{R}^{3}} \int_{\mathbb{R}^{3}}\left\{\frac{1}{2 m}\left|q+\frac{m \omega_{c}}{2}\langle\theta\rangle{ }^{\perp} x\right|^{2}+\frac{m \omega_{c}^{2}}{4}\left(\left\langle\theta^{2}\right\rangle-\langle\theta\rangle^{2}\right) \frac{\left.\left.\right|^{\perp} x\right|^{2}}{2}\right\} g^{0}(t, x, q) \mathrm{d} q \mathrm{~d} x .
$$

Moreover, when the electric potential solves the Poisson equation corresponding to the concentration

$$
\int_{\mathbb{R}^{3}} f^{0}(t, t / \varepsilon, x, p) \mathrm{d} p=\int_{\mathbb{R}^{3}} g^{0}\left(t, x, p-\frac{m \omega_{c}}{2} \theta(t / \varepsilon)^{\perp} x\right) \mathrm{d} p=\int_{\mathbb{R}^{3}} g^{0}(t, x, q) \mathrm{d} q
$$


the total energy is preserved. In order to simplify our computations we work with smooth solutions.

Proposition 5.2 Assume that $f^{\text {in }}$ is nonnegative, $f^{\text {in }} \in W^{1, \infty}\left(\mathbb{R}^{3} \times \mathbb{R}^{3}\right)$ such that

$$
\int_{\mathbb{R}^{3}} \int_{\mathbb{R}^{3}}\left(1+\left.\left.\right|^{\perp} x\right|^{2}+|p|^{2}\right) f^{\text {in }}(x, p) \mathrm{d} p \mathrm{~d} x<+\infty .
$$

i) If $E \in L_{\mathrm{loc}}^{1}\left(\mathbb{R}_{+} ; W^{1, \infty}\left(\mathbb{R}^{3}\right)\right)$ is a given electric field then

$$
\int_{\mathbb{R}^{3}} \int_{\mathbb{R}^{3}} \frac{|p|^{2}}{2 m} f^{0}(t, t / \varepsilon, x, p) \mathrm{d} p \mathrm{~d} x \rightarrow W^{0}(t) \text { weakly } \star \text { in } L_{\mathrm{loc}}^{\infty}\left(\mathbb{R}_{+}\right) \text {as } \varepsilon \searrow 0 .
$$

ii) If $f^{\text {in }}$ has compact support and $E \in L_{\text {loc }}^{1}\left(\mathbb{R}_{+} ; W^{1, \infty}\left(\mathbb{R}^{3}\right)\right)$, then there is a continuous nondecreasing function $R$ depending on $t$, such that

$$
\operatorname{supp} g^{0}(t, \cdot, \cdot) \subset\{(x, q):|x| \leq R(t),|q| \leq R(t)\}, \quad t \in \mathbb{R}_{+} .
$$

iii) If $E$ belongs to $\in L_{\text {loc }}^{1}\left(\mathbb{R}_{+} ; W^{1, \infty}\left(\mathbb{R}^{3}\right)\right)$ and solves the Poisson equation

$$
E=-\nabla_{x} \phi, \quad-\Delta_{x} \phi(t)=\frac{e}{\varepsilon_{0}}\left(\int_{\mathbb{R}^{3}} g^{0}(t, x, q) \mathrm{d} q-n_{0}(x)\right)
$$

such that $E(0, \cdot) \in\left(L^{2}\left(\mathbb{R}^{3}\right)\right)^{3}$, then

$$
\frac{d}{d t}\left\{W^{0}+\frac{\varepsilon_{0}}{2} \int_{\mathbb{R}^{3}}|E|^{2} \mathrm{~d} x\right\}=0
$$

Proof. i) It is easily seen that

$$
\begin{aligned}
\int_{\mathbb{R}^{3}} \int_{\mathbb{R}^{3}} f^{0}(t, t / \varepsilon, x, p) \mathrm{d} p \mathrm{~d} x & =\int_{\mathbb{R}^{3}} \int_{\mathbb{R}^{3}} g^{0}\left(t, x, p-\frac{m \omega_{c}}{2} \theta(t / \varepsilon)^{\perp} x\right) \mathrm{d} p \mathrm{~d} x \\
& =\int_{\mathbb{R}^{3}} \int_{\mathbb{R}^{3}} g^{0}(t, x, q) \mathrm{d} q \mathrm{~d} x \\
& =\int_{\mathbb{R}^{3}} \int_{\mathbb{R}^{3}} g^{0}(0, x, q) \mathrm{d} q \mathrm{~d} x \\
& =\int_{\mathbb{R}^{3}} \int_{\mathbb{R}^{3}} f^{\text {in }}\left(x, q+\frac{m \omega_{c}}{2} \theta(0){ }^{\perp} x\right) \mathrm{d} q \mathrm{~d} x \\
& =\int_{\mathbb{R}^{3}} \int_{\mathbb{R}^{3}} f^{\text {in }}(x, p) \mathrm{d} p \mathrm{~d} x
\end{aligned}
$$

saying that $f^{0}(t, t / \varepsilon, \cdot, \cdot)$ belongs to $L^{1}\left(\mathbb{R}_{x}^{3} \times \mathbb{R}_{p}^{3}\right)$ uniformly in $t \in \mathbb{R}_{+}, \varepsilon>0$. We consider the function

$$
\begin{aligned}
\chi(x, q) & =\frac{1}{T} \int_{0}^{T} \frac{1}{2 m}\left|q+\frac{m \omega_{c}}{2} \theta(s){ }^{\perp} x\right|^{2} \mathrm{~d} s \\
& =\frac{|q|^{2}}{2 m}+\frac{m \omega_{c}^{2}}{4}\left\langle\theta^{2}\right\rangle \frac{\left.\left.\right|^{\perp} x\right|^{2}}{2}+\frac{\omega_{c}}{2}\langle\theta\rangle\left(q \cdot{ }^{\perp} x\right) \\
& =\frac{1}{2 m}\left|q+\frac{m \omega_{c}}{2}\langle\theta\rangle{ }^{\perp} x\right|^{2}+\frac{m \omega_{c}^{2}}{4}\left(\left\langle\theta^{2}\right\rangle-\langle\theta\rangle^{2}\right) \frac{\left.\left.\right|^{\perp} x\right|^{2}}{2} .
\end{aligned}
$$


Observe that

$$
\left(\frac{q}{m}+\frac{\omega_{c}}{2}\langle\theta\rangle^{\perp} x\right) \cdot \nabla_{x} \chi+\left(e E+\frac{\omega_{c}}{2}\langle\theta\rangle^{\perp} q+\frac{m \omega_{c}^{2}}{4}\left\langle\theta^{2}\right\rangle{ }^{\perp \perp} x\right) \cdot \nabla_{q} \chi=e E \cdot\left(\frac{q}{m}+\frac{\omega_{c}}{2}\langle\theta\rangle^{\perp} x\right)
$$

and thus multiplying (34) by $\chi$ we deduce

$$
\begin{aligned}
\frac{d}{d t} \int_{\mathbb{R}^{3}} \int_{\mathbb{R}^{3}} g^{0} \chi(x, q) \mathrm{d} q \mathrm{~d} x & =\int_{\mathbb{R}^{3}} \int_{\mathbb{R}^{3}} g^{0}(t, x, q) e E \cdot\left(\frac{q}{m}+\frac{\omega_{c}}{2}\langle\theta\rangle{ }^{\perp} x\right) \mathrm{d} q \mathrm{~d} x \\
& \leq \int_{\mathbb{R}^{3}} \int_{\mathbb{R}^{3}} g^{0}|e E|\left\{\frac{1}{2 m}\left|q+\frac{m \omega_{c}}{2}\langle\theta\rangle^{\perp} x\right|^{2}+\frac{1}{2 m}\right\} \mathrm{d} q \mathrm{~d} x \\
& \leq\|e E(t)\|_{L^{\infty}\left(\mathbb{R}^{3}\right)} \int_{\mathbb{R}^{3}} \int_{\mathbb{R}^{3}} g^{0} \chi \mathrm{d} q \mathrm{~d} x+\frac{\|e E(t)\|_{L^{\infty}\left(\mathbb{R}^{3}\right)}}{2 m} \int_{\mathbb{R}^{3}} \int_{\mathbb{R}^{3}} f^{\text {in } \mathrm{d} p \mathrm{~d} x .}
\end{aligned}
$$

By Gronwall's lemma it follows that $\int_{\mathbb{R}^{3}} \int_{\mathbb{R}^{3}} g^{0}(\cdot, x, q) \chi(x, q) \mathrm{d} q \mathrm{~d} x \in L_{\text {loc }}^{\infty}\left(\mathbb{R}_{+}\right)$

$$
\begin{aligned}
\int_{\mathbb{R}^{3}} \int_{\mathbb{R}^{3}} g^{0}(t, x, q) \chi \mathrm{d} q \mathrm{~d} x & \leq\left(\int_{\mathbb{R}^{3}} \int_{\mathbb{R}^{3}} g^{0}(0, x, q) \chi \mathrm{d} q \mathrm{~d} x+\frac{\|e E\|_{L^{1}\left([0, t] ; L^{\infty}\left(\mathbb{R}^{3}\right)\right)}}{2 m} \int_{\mathbb{R}^{3}} \int_{\mathbb{R}^{3}} f^{\text {in }} \mathrm{d} p \mathrm{~d} x\right) \\
& \times \exp \left(\int_{0}^{t}\|e E(s)\|_{L^{\infty}\left(\mathbb{R}^{3}\right)} \mathrm{d} s\right) .
\end{aligned}
$$

For any function $\eta \in L_{\text {loc }}^{1}\left(\mathbb{R}_{+}\right)$we can write

$$
\begin{aligned}
& \lim _{\varepsilon \searrow 0} \int_{\mathbb{R}_{+}} \eta(t) \int_{\mathbb{R}^{3}} \int_{\mathbb{R}^{3}} f^{0}(t, t / \varepsilon, x, p) \frac{|p|^{2}}{2 m} \mathrm{~d} p \mathrm{~d} x \mathrm{~d} t \\
= & \lim _{\varepsilon \searrow 0} \int_{\mathbb{R}_{+}} \eta(t) \int_{\mathbb{R}^{3}} \int_{\mathbb{R}^{3}} g^{0}\left(t, x, p-\frac{m \omega_{c}}{2} \theta(t / \varepsilon)^{\perp} x\right) \frac{|p|^{2}}{2 m} \mathrm{~d} p \mathrm{~d} x \mathrm{~d} t \\
= & \lim _{\varepsilon \searrow 0} \int_{\mathbb{R}_{+}} \eta(t) \int_{\mathbb{R}^{3}} \int_{\mathbb{R}^{3}} g^{0}(t, x, q) \frac{1}{2 m}\left|q+\frac{m \omega_{c}}{2} \theta(t / \varepsilon)^{\perp} x\right|^{2} \mathrm{~d} q \mathrm{~d} x \mathrm{~d} t \\
= & \int_{\mathbb{R}_{+}} \eta(t) \int_{\mathbb{R}^{3}} \int_{\mathbb{R}^{3}} g^{0}(t, x, q) \chi(x, q) \mathrm{d} q \mathrm{~d} x \mathrm{~d} t
\end{aligned}
$$

saying that

$$
\int_{\mathbb{R}^{3}} \int_{\mathbb{R}^{3}} \frac{|p|^{2}}{2 m} f^{0}(t, t / \varepsilon, x, p) \mathrm{d} p \mathrm{~d} x \rightarrow W^{0}(t) \text { weakly } \star \text { in } L_{\text {loc }}^{\infty}\left(\mathbb{R}_{+}\right) \text {as } \varepsilon \searrow 0 .
$$

ii) Assume that supp $f^{\text {in }} \subset\left\{(x, p):|x| \leq R^{\text {in }},|p| \leq R^{\text {in }}\right\}$ with $R^{\text {in }}>0$. Therefore

$$
\begin{aligned}
\operatorname{supp} g^{0}(0, \cdot, \cdot) & \subset\left\{(x, q):|x| \leq R^{\text {in }},|q| \leq\left(1+m\left|\omega_{c}\right| / 2\|\theta\|_{L^{\infty}\left(\mathbb{R}^{3}\right)}\right) R^{\text {in }}\right\} \\
& \subset\left\{(x, q): \sqrt{\chi(x, q)} \leq \nu R^{\text {in }},\left|x_{3}\right| \leq \nu R^{\text {in }}\right\}
\end{aligned}
$$

for some $\nu>0$. We consider a function $\xi$ satisfying

$$
\xi \in C^{1}(\mathbb{R}), \quad 0 \leq \xi \leq 1, \quad \operatorname{supp} \xi=\mathbb{R}_{+}, \quad \xi^{\prime} \geq 0
$$


Applying the weak formulation of (34) with the test function $(t, x, q) \rightarrow \xi(\sqrt{\chi(x, q)}-$ $\alpha(t))$, with $\alpha \in C^{1}\left(\mathbb{R}_{+}\right)$yields by (43)

$$
\begin{aligned}
& \int_{\mathbb{R}^{3}} \int_{\mathbb{R}^{3}} g^{0}(t, x, q) \xi(\sqrt{\chi(x, q)}-\alpha(t)) \mathrm{d} q \mathrm{~d} x-\int_{\mathbb{R}^{3}} \int_{\mathbb{R}^{3}} g^{0}(0, x, q) \xi(\sqrt{\chi(x, q)}-\alpha(0)) \mathrm{d} q \mathrm{~d} x \\
= & \int_{0}^{t} \int_{\mathbb{R}^{3}} \int_{\mathbb{R}^{3}} g^{0}(\tau, x, q) \xi^{\prime}\left\{-\alpha^{\prime}(\tau)+\frac{e E(\tau, x)}{2 \sqrt{\chi(x, q)}} \cdot\left(\frac{q}{m}+\frac{\omega_{c}}{2}\langle\theta\rangle{ }^{\perp} x\right)\right\} \mathrm{d} q \mathrm{~d} x \mathrm{~d} \tau \\
\leq & \int_{0}^{t} \int_{\mathbb{R}^{3}} \int_{\mathbb{R}^{3}} g^{0}(\tau, x, q) \xi^{\prime}\left\{-\alpha^{\prime}(\tau)+\frac{\|e E(\tau)\|_{L^{\infty}\left(\mathbb{R}^{3}\right)}}{\sqrt{2 m}}\right\} \mathrm{d} q \mathrm{~d} x \mathrm{~d} \tau .
\end{aligned}
$$

We take $\alpha(0)=\nu R^{\text {in }}$ and $\alpha^{\prime}(\tau)=\frac{\|e E(\tau)\|_{L^{\infty}\left(\mathbb{R}^{3}\right)}}{\sqrt{2 m}}, \tau \in \mathbb{R}_{+}$. Since $g^{0}(0, x, q) \xi(\sqrt{\chi(x, q)}-$ $\alpha(0))=0$ one gets

$$
\int_{\mathbb{R}^{3}} \int_{\mathbb{R}^{3}} g^{0}(t, x, q) \xi(\sqrt{\chi(x, q)}-\alpha(t)) \mathrm{d} q \mathrm{~d} x \leq 0
$$

implying that supp $g^{0}(t, \cdot, \cdot) \subset\{(x, q): \sqrt{\chi(x, q)} \leq \alpha(t)\}$. Similarly, applying the weak formulation of (34) with the test function $(t, x, q) \rightarrow \xi\left(\left|x_{3}-t q_{3} / m\right|-\beta(t)\right), \beta \in C^{1}\left(\mathbb{R}_{+}\right)$ we obtain

$$
\begin{aligned}
& \int_{\mathbb{R}^{3}} \int_{\mathbb{R}^{3}} g^{0}(t, x, q) \xi\left(\left|x_{3}-t q_{3} / m\right|-\beta(t)\right) \mathrm{d} q \mathrm{~d} x-\int_{\mathbb{R}^{3}} \int_{\mathbb{R}^{3}} g^{0}(0, x, q) \xi\left(\left|x_{3}\right|-\beta(0)\right) \mathrm{d} q \mathrm{~d} x \\
= & \int_{0}^{t} \int_{\mathbb{R}^{3}} \int_{\mathbb{R}^{3}} g^{0}(\tau, x, q) \xi^{\prime}\left\{-\beta^{\prime}(\tau)-\frac{\tau e E_{3}(\tau, x)}{m} \operatorname{sgn}\left(x_{3}-t q_{3} / m\right)\right\} \mathrm{d} q \mathrm{~d} x \mathrm{~d} \tau \\
\leq & \int_{0}^{t} \int_{\mathbb{R}^{3}} \int_{\mathbb{R}^{3}} g^{0}(\tau, x, q) \xi^{\prime}\left\{-\beta^{\prime}(\tau)+\frac{\tau\left\|e E_{3}(\tau)\right\|_{L^{\infty}\left(\mathbb{R}^{3}\right)}}{m}\right\} \mathrm{d} q \mathrm{~d} x \mathrm{~d} \tau .
\end{aligned}
$$

Taking $\beta(0)=\nu R^{\text {in }}$ and $\beta^{\prime}(\tau)=\frac{\tau\left\|e E_{3}(\tau)\right\|_{L^{\infty}\left(\mathbb{R}^{3}\right)}}{m}, \tau \in \mathbb{R}_{+}$we deduce that

$$
\operatorname{supp} g^{0}(t, \cdot, \cdot) \subset\left\{(x, q):\left|x_{3}-t q_{3} / m\right| \leq \beta(t)\right\} \text {. }
$$

We have proved that

$$
\begin{aligned}
\operatorname{supp} g^{0}(t) & \subset\left\{(x, q): \sqrt{\chi(x, q)} \leq \nu R^{\text {in }}+\frac{|e|}{\sqrt{2 m}}\|E\|_{L^{1}\left([0, t] ; L^{\infty}\left(\mathbb{R}^{3}\right)\right)}\right\} \\
& \cap\left\{(x, q):\left|x_{3}\right| \leq \nu R^{\text {in }}+t \frac{\left|q_{3}\right|}{m}+\frac{\left.t\left\|e E_{3}\right\|_{L^{1}\left([0, t] ; L^{\infty}\left(\mathbb{R}^{3}\right)\right)}\right\}}{m}\right\} \\
& \subset\{(x, q):|x| \leq R(t),|q| \leq R(t)\}
\end{aligned}
$$

for some continuous nondecreasing function $R(t)$. Notice also that for any $t \in \mathbb{R}_{+}$

$$
\operatorname{supp} f^{0}(t, \cdot, \cdot, \cdot) \subset\left\{(s, x, p):|x| \leq R(t), \quad|p| \leq\left(1+m\left|\omega_{c}\right| / 2\|\theta\|_{L^{\infty}\left(\mathbb{R}^{3}\right)}\right) R(t)\right\} .
$$


iii) Assume now that the electric potential solves the Poisson equation (42). By standard computations involving the continuity equation

$$
\partial_{t} \int_{\mathbb{R}^{3}} g^{0} \mathrm{~d} q+\operatorname{div}_{x} \int_{\mathbb{R}^{3}}\left(\frac{q}{m}+\frac{\omega_{c}}{2}\langle\theta\rangle^{\perp} x\right) g^{0} \mathrm{~d} q=0
$$

one gets

$$
\frac{d}{d t} \frac{\varepsilon_{0}}{2} \int_{\mathbb{R}^{3}}|E|^{2} \mathrm{~d} x=-\int_{\mathbb{R}^{3}} e E(t, x) \cdot \int_{\mathbb{R}^{3}}\left(\frac{q}{m}+\frac{\omega_{c}}{2}\langle\theta\rangle{ }^{\perp} x\right) g^{0} \mathrm{~d} q \mathrm{~d} x .
$$

Combining (44), (46) yields

$$
\frac{d}{d t}\left\{\int_{\mathbb{R}^{3}} \int_{\mathbb{R}^{3}} g^{0}(t, x, q) \chi(x, q) \mathrm{d} q \mathrm{~d} x+\frac{\varepsilon_{0}}{2} \int_{\mathbb{R}^{3}}|E(t, x)|^{2} \mathrm{~d} x\right\}=0 .
$$

In the sequel we inquire about the confinement properties of the limit model (34), (40) (resp. (13), (14)). We exploit here the invariants of (34). Let us consider for the moment that the potential is stationary. In this case notice that (34) writes under the Hamiltonian form

$$
\partial_{t} g^{0}+\nabla_{q} H \cdot \nabla_{x} g^{0}-\nabla_{x} H \cdot \nabla_{q} g^{0}=0
$$

with the Hamiltonian

$$
H(x, q)=\chi(x, q)+e \phi(x)=\frac{1}{2 m}\left|q+\frac{m \omega_{c}}{2}\langle\theta\rangle^{\perp} x\right|^{2}+\frac{m \omega_{c}^{2}}{4}\left(\left\langle\theta^{2}\right\rangle-\langle\theta\rangle^{2}\right) \frac{\left.\left.\right|^{\perp} x\right|^{2}}{2}+e \phi(x) .
$$

In particular $H$ is a stationary solution of (34) or equivalently $H$ is an invariant of the characteristic flow of (34)

$$
\frac{d X}{d t}=\nabla_{q} H(X(t), Q(t)), \quad \frac{d Q}{d t}=-\nabla_{x} H(X(t), Q(t)) .
$$

Under additional hypotheses on the electric potential $\phi$ it is easily seen that the plasma remains confined in a bounded region around the magnetic field lines. Indeed, assume that the hypothesis in Theorem 2.2 holds true

$$
\lim _{\left|{ }^{\perp} x\right| \rightarrow+\infty}\left\{e \phi(x)+\frac{m \omega_{c}^{2}}{4}\left(\left\langle\theta^{2}\right\rangle-\langle\theta\rangle^{2}\right) \frac{\left.\left.\right|^{\perp} x\right|^{2}}{2}\right\}=+\infty
$$

uniformly with respect to $x_{3}$ and that at the initial time we have

$$
0 \leq g^{0}(0, x, q) \leq \lambda(H(x, q)), \quad(x, q) \in \mathbb{R}^{3} \times \mathbb{R}^{3}
$$


for some nonnegative profile $\lambda \in C^{1}(\mathbb{R})$, vanishing on $[L,+\infty[$. By the maximum principle we deduce that

$$
0 \leq g^{0}(t, x, q) \leq \lambda(H(x, q)), \quad(t, x, q) \in \mathbb{R}_{+} \times \mathbb{R}^{3} \times \mathbb{R}^{3}
$$

which guarantees the compactness of the support of $g^{0}(t)$ along the orthogonal directions to the magnetic lines, uniformly in time. Indeed, we have

$\operatorname{supp} g^{0}(t) \subset\{(x, q): H(x, q) \leq L\} \subset\left\{(x, q): e \phi(x)+\frac{m \omega_{c}^{2}}{4}\left(\left\langle\theta^{2}\right\rangle-\langle\theta\rangle^{2}\right) \frac{\left.\left.\right|^{\perp} x\right|^{2}}{2} \leq L\right\}$.

By the hypothesis (47) there is $R>0$ such that for any $x \in \mathbb{R}^{3}$ verifying $\left.\right|^{\perp} x \mid>R$ we have

$$
e \phi(x)+\frac{m \omega_{c}^{2}}{4}\left(\left\langle\theta^{2}\right\rangle-\langle\theta\rangle^{2}\right) \frac{\left.\left.\right|^{\perp} x\right|^{2}}{2}>L
$$

and finally

$$
\operatorname{supp} g^{0}(t) \subset\left\{(x, q):\left.\right|^{\perp} x \mid \leq R\right\}, \quad \forall t \in \mathbb{R}_{+} .
$$

Remark 5.1 If the electric potential depends only on $\left.\right|^{\perp} x \mid$ and $x_{3}$ then $\left(q \cdot{ }^{\perp} x\right)$ is another invariant of (34). If the electric potential depends only on $\left.\right|^{\perp} x \mid$ then $q_{3}$ is an invariant of (34) as well.

When the electric potential depends on time, the previous Hamiltonian becomes $H(t, x, q)=$ $\chi(x, q)+e \phi(t, x)$ and therefore we obtain

$$
\partial_{t} H+\left(\frac{q}{m}+\frac{\omega_{c}}{2}\langle\theta\rangle^{\perp} x\right) \cdot \nabla_{x} H+\left(e E+\frac{\omega_{c}}{2}\left\langle\theta{ }^{\perp} q+\frac{m \omega_{c}^{2}}{4}\left\langle\theta^{2}\right\rangle{ }^{\perp \perp} x\right) \cdot \nabla_{q} H=e \partial_{t} \phi .\right.
$$

In this case we need to construct a particular super-solution for (34).

Proof. (of Theorem 2.2 for time dependent electric potential) We work in the phase space $(x, q)$, using the equation (34) and the initial condition (40). By the hypotheses we know that

$$
g^{0}(0, x, q) \leq \lambda(\chi(x, q)+e \phi(0, x)), \quad(x, q) \in \mathbb{R}^{3} \times \mathbb{R}^{3}
$$

Let us consider the function $h$ defined for any $(t, x, q) \in \mathbb{R}_{+} \times \mathbb{R}^{3} \times \mathbb{R}^{3}$ by

$$
h(t, x, q)=\lambda\left(\chi(x, q)+e \phi(t, x)-\int_{0}^{t} \sup _{y \in \mathbb{R}^{3}}\left\{e \partial_{t} \phi(s, y)\right\} \mathrm{d} s\right) .
$$


Observe that

$$
h(0, x, q)=\lambda(\chi(x, q)+e \phi(0, x)) \geq g^{0}(0, x, q), \quad(x, q) \in \mathbb{R}^{3} \times \mathbb{R}^{3} .
$$

Taking into account (49) it is easily seen, by the monotonicity of $\lambda$, that

$$
\partial_{t} h+\left(\frac{q}{m}+\frac{\omega_{c}}{2}\langle\theta\rangle^{\perp} x\right) \cdot \nabla_{x} h+\left(e E+\frac{\omega_{c}}{2}\left\langle\theta{ }^{\perp} q+\frac{m \omega_{c}^{2}}{4}\left\langle\theta^{2}\right\rangle^{\perp \perp} x\right) \cdot \nabla_{q} h \geq 0 .\right.
$$

By the maximum principle we deduce that

$$
g^{0}(t, x, q) \leq h(t, x, q), \quad(t, x, q) \in \mathbb{R}_{+} \times \mathbb{R}^{3} \times \mathbb{R}^{3} .
$$

By the hypothesis (16) there is $R>0$ such that for any $(t, x) \in \mathbb{R}_{+} \times \mathbb{R}^{3}$, with $\left|{ }^{\perp} x\right|>R$ we have

$$
e \phi(t, x)-\int_{0}^{t} \sup _{y \in \mathbb{R}^{3}}\left\{e \partial_{t} \phi(s, y)\right\} \mathrm{d} s+\frac{m \omega_{c}^{2}}{4}\left(\left\langle\theta^{2}\right\rangle-\langle\theta\rangle^{2}\right) \frac{\left.\left.\right|^{\perp} x\right|^{2}}{2}>L
$$

implying that for any $t \in \mathbb{R}_{+}$

$$
\begin{aligned}
\operatorname{supp} g^{0}(t) & \subset\left\{(x, q): \chi(x, q)+e \phi(t, x)-\int_{0}^{t} \sup _{y \in \mathbb{R}^{3}}\left\{e \partial_{t} \phi(s, y)\right\} \mathrm{d} s \leq L\right\} \\
& \subset\left\{(x, q): e \phi(t, x)-\int_{0}^{t} \sup _{y \in \mathbb{R}^{3}}\left\{e \partial_{t} \phi(s, y)\right\} \mathrm{d} s+\frac{m \omega_{c}^{2}}{4}\left(\left\langle\theta^{2}\right\rangle-\langle\theta\rangle^{2}\right) \frac{\left.\left.\right|^{\perp} x\right|^{2}}{2} \leq L\right\} \\
& \subset\left\{(x, q):\left.\right|^{\perp} x \mid \leq R\right\} .
\end{aligned}
$$

Since $f^{0}(t, s, x, p)=g^{0}\left(t, x, p-m \omega_{c} \theta(s) / 2{ }^{\perp} x\right)$ finally one gets

$$
\operatorname{supp} f^{0}(t, s, \cdot, \cdot) \subset\left\{(x, p):\left.\right|^{\perp} x \mid \leq R\right\}, \quad t \in \mathbb{R}_{+}, s \in \mathbb{R} \text {. }
$$

In particular

$$
\operatorname{supp} f^{0}(t, t / \varepsilon, \cdot, \cdot) \subset\left\{(x, p):\left.\right|^{\perp} x \mid \leq R\right\}, \quad t \in \mathbb{R}_{+}, \quad \varepsilon>0 .
$$

\section{Asymptotic behaviour}

The aim of this section is to justify rigorously the Hilbert expansion (17). More precisely we intend to prove that $f^{\varepsilon}(t, x, p)=f^{0}(t, t / \varepsilon, x, p)+\mathcal{O}(\varepsilon)$ strongly in $L^{2}\left(\mathbb{R}^{3} \times\right.$ 
$\mathbb{R}^{3}$ ), uniformly for $t$ in bounded intervals. The idea is to introduce the solution $F^{\varepsilon}=$ $F^{\varepsilon}(t, s, x, p)$ of the transport problem

$$
\partial_{t} F^{\varepsilon}+\frac{p}{m} \cdot \nabla_{x} F^{\varepsilon}+\left(e E(t, x)+\omega_{c} \theta(s)^{\perp} p\right) \cdot \nabla_{p} F^{\varepsilon}+\frac{1}{\varepsilon} \mathcal{T} F^{\varepsilon}=0
$$

with the initial condition

$$
F^{\varepsilon}(0, s, x, p)=f^{\text {in }}\left(x, p-\frac{m \omega_{c}}{2} \theta(s)^{\perp} x+\frac{m \omega_{c}}{2} \theta(0)^{\perp} x\right)
$$

and to observe that $F^{\varepsilon}(t, t / \varepsilon, x, p)$ satisfies (5), (2), saying that $F^{\varepsilon}(t, t / \varepsilon, x, p)=$ $f^{\varepsilon}(t, x, p)$. We start by estimating the error between $F^{\varepsilon}(t, s, x, p)$ and $f^{0}(t, s, x, p)$. We also prove that for any $I \in \mathbb{R}_{+}$the functions $\left\{F^{\varepsilon}(t): t \in[0, I], \varepsilon>0\right\}$ are uniformly compactly supported.

Proposition 6.1 Assume that $E \in L_{\mathrm{loc}}^{1}\left(\mathbb{R}_{+} ; W^{2, \infty}\left(\mathbb{R}^{3}\right)\right), \partial_{t} E \in L_{\mathrm{loc}}^{1}\left(\mathbb{R}_{+} ; L^{\infty}\left(\mathbb{R}^{3}\right)\right)$ for any $I \in \mathbb{R}_{+}, f^{\text {in }} \in W^{2, \infty}\left(\mathbb{R}^{3} \times \mathbb{R}^{3}\right)$ and supp $f^{\text {in }}$ is compact. Then for any $I \in \mathbb{R}_{+}$, there is a constant $C_{1}(I)$ such that

$$
\left\|F^{\varepsilon}(t)-f^{0}(t)\right\| \leq C_{1}(I) \varepsilon, \quad t \in I, \quad \varepsilon>0 .
$$

Proof. By Proposition 5.2 ii), the solution of the problem (13), (14) has compact support, uniformly for $t$ in bounded intervals i.e., $\forall I \in \mathbb{R}_{+}, \exists R(I)$ such that

$$
\operatorname{supp} f^{0}(t, \cdot, \cdot, \cdot) \subset\{(s, x, p):|x| \leq R(I), \quad|p| \leq R(I)\}, \quad t \in[0, I] .
$$

Under our hypotheses, the solution $f^{0}$ has the regularity

$$
f^{0}, \nabla_{(t, x, p)} f^{0}, \nabla_{(t, x, p)}^{2} f^{0} \in L^{\infty}\left([0, I] \times \mathbb{R}_{s} \times \mathbb{R}^{3} \times \mathbb{R}^{3}\right), \quad I \in \mathbb{R}_{+} .
$$

Recall that the model (35) is equivalent to (33). By Proposition 3.2, for any $t \in \mathbb{R}_{+}$, there is a unique function $f^{1}(t)$ of zero average such that

$$
\partial_{t} f^{0}+\frac{p}{m} \cdot \nabla_{x} f^{0}+\left(e E(t, x)+\omega_{c} \theta(s)^{\perp} p\right) \cdot \nabla_{p} f^{0}+\mathcal{T} f^{1}(t)=0 .
$$

Since $f^{0}$ is smooth and has compact support (uniformly with respect to $t$ in bounded intervals) the following set

$$
\left\{\nabla_{(t, x, p)}\left(\partial_{t} f^{0}+\frac{p}{m} \cdot \nabla_{x} f^{0}+\left(e E(t, x)+\omega_{c} \theta(s){ }^{\perp} p\right) \cdot \nabla_{p} f^{0}\right): t \in[0, I]\right\}
$$


is bounded in $L_{\#}^{2}\left(\mathbb{R}_{s} ; L^{2}\left(\mathbb{R}_{x}^{3} \times \mathbb{R}_{p}^{3}\right)\right)$. By Propositions $4.4,4.7$ we deduce that

$$
\left\{\nabla_{(t, x, p)} f^{1}(t): t \in[0, I]\right\}
$$

remains bounded in $L_{\#}^{2}\left(\mathbb{R}_{s} ; L^{2}\left(\mathbb{R}_{x}^{3} \times \mathbb{R}_{p}^{3}\right)\right)$. Combining (50), (52) and the constraint (31) yields

$$
\begin{aligned}
& \left(\partial_{t}+\frac{p}{m} \cdot \nabla_{x}+\left(e E(t, x)+\omega_{c} \theta(s){ }^{\perp} p\right) \cdot \nabla_{p}\right)\left\{F^{\varepsilon}-f^{0}-\varepsilon f^{1}\right\}+\frac{1}{\varepsilon} \mathcal{T}\left\{F^{\varepsilon}-f^{0}-\varepsilon f^{1}\right\} \\
& =-\varepsilon\left\{\partial_{t} f^{1}+\frac{p}{m} \cdot \nabla_{x} f^{1}+\left(e E(t, x)+\omega_{c} \theta(s){ }^{\perp} p\right) \cdot \nabla_{p} f^{1}\right\}
\end{aligned}
$$

and after integration with respect to $(s, x, p)$ one gets

$$
\frac{d}{d t}\left\|F^{\varepsilon}-f^{0}-\varepsilon f^{1}\right\| \leq \varepsilon\left\|\partial_{t} f^{1}+\frac{p}{m} \cdot \nabla_{x} f^{1}+\left(e E(t)+\omega_{c} \theta^{\perp} p\right) \cdot \nabla_{p} f^{1}\right\| .
$$

Taking into account that $F^{\varepsilon}, f^{0}$ satisfy the same initial condition we deduce that $\left\|F^{\varepsilon}(t)-f^{0}(t)-\varepsilon f^{1}(t)\right\| \leq \varepsilon\left\|f^{1}(0)\right\|+\varepsilon \int_{0}^{t}\left\|\left(\partial_{t}+\frac{p}{m} \cdot \nabla_{x}+\left(e E(r)+\omega_{c} \theta^{\perp} p\right) \cdot \nabla_{p}\right) f^{1}\right\| \mathrm{d} r$ and finally

$$
\left\|F^{\varepsilon}(t)-f^{0}(t)\right\| \leq C_{1}(I) \varepsilon, \quad t \in I, \quad \varepsilon>0 .
$$

Proposition 6.2 Assume that the electric field is smooth $E \in L_{\text {loc }}^{1}\left(\mathbb{R}_{+} ; W^{1, \infty}\left(\mathbb{R}^{3}\right)\right)$ and that $f^{\text {in }}$ has compact support supp $f^{\text {in }} \subset\left\{(x, p):|x| \leq R^{\text {in }},|p| \leq R^{\text {in }}\right\}$. Then there is a continuous nondecreasing function $\delta: \mathbb{R}_{+} \rightarrow \mathbb{R}_{+}$such that

$$
\operatorname{supp} F^{\varepsilon}(t, \cdot, \cdot, \cdot) \subset\left\{(s, x, p):|p| \leq \delta(t), \frac{m\left|\omega_{c}\right|\|\theta\|_{L^{\infty}}}{2}|x| \leq \delta(t)\right\}, t \in \mathbb{R}_{+}, \quad \varepsilon>0 .
$$

In particular for any $I \in \mathbb{R}_{+}$

$$
\bigcup_{\varepsilon>0, t \in[0, I]} \operatorname{supp} F^{\varepsilon}(t, \cdot, \cdot, \cdot) \subset\left\{(s, x, p):|p| \leq \delta(I), \frac{m\left|\omega_{c}\right|\|\theta\|_{L^{\infty}}}{2}|x| \leq \delta(I)\right\} .
$$

Proof. Let us consider a function $\xi \in C^{1}(\mathbb{R})$ satisfying

$$
0 \leq \xi \leq 1, \quad \xi^{\prime} \geq 0, \quad \operatorname{supp} \xi=\mathbb{R}_{+}, \quad z \xi^{\prime}(z) \leq C \xi(z), \quad z \in \mathbb{R}
$$

for some constant $C>0$. We denote by $h$ the function

$$
h(s, x, p)=\left|p-\frac{m \omega_{c}}{2} \theta(s)^{\perp} x\right|+\frac{m\left|\omega_{c}\right|}{2}\|\theta\|_{L^{\infty}}|x| .
$$


Notice that $h$ depends only on the invariants $x$ and $q=p-\frac{m \omega_{c}}{2} \theta(s){ }^{\perp} x$ and therefore $\mathcal{T} h=0$. By direct computations one gets

$$
\nabla_{x}\left|p-\frac{m \omega_{c}}{2} \theta(s){ }^{\perp} x\right|=\frac{m \omega_{c}}{2} \theta(s) \frac{{ }^{\perp} q}{|q|}, \quad \nabla_{p}\left|p-\frac{m \omega_{c}}{2} \theta(s)^{\perp} x\right|=\frac{q}{|q|}
$$

and therefore

$$
\begin{aligned}
\left(\frac{p}{m} \cdot \nabla_{x}+\left(e E+\omega_{c} \theta(s){ }^{\perp} p\right) \cdot \nabla_{p}\right)\left|p-\frac{m \omega_{c}}{2} \theta(s){ }^{\perp} x\right| & =e E(t) \cdot \frac{q}{|q|}+\frac{m \omega_{c}^{2} \theta^{2}(s)}{4}{ }^{\perp \perp} x \cdot \frac{q}{|q|} \\
& \leq\|e E(t)\|_{L^{\infty}\left(\mathbb{R}^{3}\right)}+\frac{m \omega_{c}^{2}}{4}\|\theta\|_{L^{\infty}}^{2}|x| .
\end{aligned}
$$

Similarly

$$
\begin{aligned}
\left(\frac{p}{m} \cdot \nabla_{x}+\left(e E(t, x)+\omega_{c} \theta(s){ }^{\perp} p\right) \cdot \nabla_{p}\right) \frac{m\left|\omega_{c}\right|\|\theta\|_{L^{\infty}}}{2}|x| & =\frac{p}{m} \cdot \frac{m\left|\omega_{c}\right|\|\theta\|_{L^{\infty}}}{2} \frac{x}{|x|} \\
& \leq \frac{\left|\omega_{c}\right|\|\theta\|_{L^{\infty}}}{2}|q| .
\end{aligned}
$$

Finally the function $h$ satisfies

$$
\left(\partial_{t}+\frac{p}{m} \cdot \nabla_{x}+\left(e E(t, x)+\omega_{c} \theta(s)^{\perp} p\right) \cdot \nabla_{p}+\frac{1}{\varepsilon} \mathcal{T}\right) h \leq\|e E(t)\|_{L^{\infty}\left(\mathbb{R}^{3}\right)}+\frac{\left|\omega_{c}\right|\|\theta\|_{L^{\infty}}}{2} h .
$$

Using now the weak formulation of (50) with the test function $(t, s, x, p) \rightarrow \xi(h(s, x, p)-$ $\delta(t))$, with $\delta \in C^{1}\left(\mathbb{R}_{+}\right)$yields

$$
\begin{aligned}
& \int_{0}^{T} \int_{\mathbb{R}^{3}} \int_{\mathbb{R}^{3}} F^{\varepsilon}(t) \xi(h(s, x, p)-\delta(t)) \mathrm{d} p \mathrm{~d} x \mathrm{~d} s-\int_{0}^{T} \int_{\mathbb{R}^{3}} \int_{\mathbb{R}^{3}} F^{\varepsilon}(0) \xi(h(s, x, p)-\delta(0)) \mathrm{d} p \mathrm{~d} x \mathrm{~d} s \\
\leq & \int_{0}^{t} \int_{0}^{T} \int_{\mathbb{R}^{3}} \int_{\mathbb{R}^{3}} F^{\varepsilon}(\tau) \xi^{\prime}(h(s, x, p)-\delta(\tau))\left\{-\delta^{\prime}(\tau)+\|e E(\tau)\|_{L^{\infty}}+\frac{\left|\omega_{c}\right|\|\theta\|_{L^{\infty}}}{2} h\right\} \mathrm{d} p \mathrm{~d} x \mathrm{~d} s \mathrm{~d} \tau .
\end{aligned}
$$

Notice that

$$
\operatorname{supp} F^{\varepsilon}(0, \cdot, \cdot, \cdot) \subset\left\{(s, x, p):|x| \leq R^{\text {in }},|p| \leq\left(1+m\left|\omega_{c}\right|\|\theta\|_{L^{\infty}}\right) R^{\text {in }}\right\}
$$

and therefore $\delta_{0}:=\sup \left\{h(s, x, p):(s, x, p) \in \cup_{\varepsilon>0} \operatorname{supp} F^{\varepsilon}(0)\right\}<+\infty$. In this case we have

$$
\int_{0}^{T} \int_{\mathbb{R}^{3}} \int_{\mathbb{R}^{3}} F^{\varepsilon}(0, s, x, p) \xi\left(h(s, x, p)-\delta_{0}\right) \mathrm{d} p \mathrm{~d} x \mathrm{~d} s=0, \quad \varepsilon>0 .
$$

We determine the function $\delta$ by solving

$$
\delta^{\prime}(\tau)=\|e E(\tau)\|_{L^{\infty}}+\frac{\left|\omega_{c}\right|\|\theta\|_{L^{\infty}}}{2} \delta(\tau), \quad \tau \in \mathbb{R}_{+}
$$


with the initial condition $\delta(0)=\delta_{0}$. The right hand side of (53) becomes

$$
\begin{aligned}
& \int_{0}^{t} \int_{0}^{T} \int_{\mathbb{R}^{3}} \int_{\mathbb{R}^{3}} F^{\varepsilon}(\tau) \xi^{\prime}(h(s, x, p)-\delta(\tau)) \frac{\left|\omega_{c}\right|\|\theta\|_{L^{\infty}}}{2}(h-\delta(\tau)) \mathrm{d} p \mathrm{~d} x \mathrm{~d} s \mathrm{~d} \tau \\
\leq & C \frac{\left|\omega_{c}\right|\|\theta\|_{L^{\infty}}}{2} \int_{0}^{t} \int_{0}^{T} \int_{\mathbb{R}^{3}} \int_{\mathbb{R}^{3}} F^{\varepsilon}(\tau) \xi(h(s, x, p)-\delta(\tau)) \mathrm{d} p \mathrm{~d} x \mathrm{~d} s \mathrm{~d} \tau .
\end{aligned}
$$

Combining (53), (54), (55) implies

$\int_{0}^{T} \int_{\mathbb{R}^{3}} \int_{\mathbb{R}^{3}} F^{\varepsilon}(t) \xi(h-\delta(t)) \mathrm{d} p \mathrm{~d} x \mathrm{~d} s \leq C \frac{\left|\omega_{c}\right|\|\theta\|_{L^{\infty}}}{2} \int_{0}^{t} \int_{0}^{T} \int_{\mathbb{R}^{3}} \int_{\mathbb{R}^{3}} F^{\varepsilon}(\tau) \xi(h-\delta(\tau)) \mathrm{d} p \mathrm{~d} x \mathrm{~d} s \mathrm{~d} \tau$ and by Gronwall's lemma we deduce that

$$
\int_{0}^{T} \int_{\mathbb{R}^{3}} \int_{\mathbb{R}^{3}} F^{\varepsilon}(t, s, x, p) \xi(h(s, x, p)-\delta(t)) \mathrm{d} p \mathrm{~d} x \mathrm{~d} s=0, \quad t \in \mathbb{R}_{+}
$$

and therefore

$\operatorname{supp} F^{\varepsilon}(t) \subset\{(s, x, p): h(s, x, p) \leq \delta(t)\} \subset\left\{(s, x, p):|p| \leq \delta(t), \frac{m\left|\omega_{c}\right|\|\theta\|_{L^{\infty}}}{2}|x| \leq \delta(t)\right\}$.

Once we have estimated the error between $F^{\varepsilon}$ and $f^{0}$, the asymptotic behaviour of $f^{\varepsilon}(t, x, p)-f^{0}(t, t / \varepsilon, x, p)$ as $\varepsilon \searrow 0$ follows by using the Sobolev inequality in Proposition 3.3 .

Proof. (of Theorem 2.3) By Proposition 3.3 we have

$$
\begin{aligned}
\left\|f^{\varepsilon}(t, \cdot, \cdot)-f^{0}(t, t / \varepsilon, \cdot, \cdot)\right\|_{L^{2}\left(\mathbb{R}^{3} \times \mathbb{R}^{3}\right)} & =\left\|F^{\varepsilon}(t, t / \varepsilon, \cdot, \cdot)-f^{0}(t, t / \varepsilon, \cdot, \cdot)\right\|_{L^{2}\left(\mathbb{R}^{3} \times \mathbb{R}^{3}\right)} \\
& \leq\left\|F^{\varepsilon}(t, \cdot, \cdot, \cdot)-f^{0}(t, \cdot, \cdot, \cdot)\right\|_{L_{\#}^{\infty}\left(\mathbb{R}_{s} ; L^{2}\left(\mathbb{R}_{x}^{3} \times \mathbb{R}_{p}^{3}\right)\right)} \\
& \leq C(T)\left(\left\|F^{\varepsilon}(t)-f^{0}(t)\right\|+\left\|\mathcal{T} F^{\varepsilon}(t)\right\|\right)
\end{aligned}
$$

since $\mathcal{T} f^{0}(t)=0$ for any $t \in \mathbb{R}_{+}$. Thanks to Proposition 6.1 we know that

$$
\left\|F^{\varepsilon}(t)-f^{0}(t)\right\| \leq C_{1}(I) \varepsilon, \quad t \in[0, I], \quad \varepsilon>0
$$

and we are done if we can find a constant $C_{2}(I)$ such that

$$
\left\|\mathcal{T} F^{\varepsilon}(t)\right\| \leq C_{2}(I) \varepsilon, \quad t \in[0, I], \quad \varepsilon>0
$$

since in that case we would obtain

$$
\left\|f^{\varepsilon}(t, \cdot, \cdot)-f^{0}(t, t / \varepsilon, \cdot, \cdot)\right\|_{L^{2}\left(\mathbb{R}^{3} \times \mathbb{R}^{3}\right)} \leq C(T)\left(C_{1}(I)+C_{2}(I)\right) \varepsilon, \quad t \in[0, I], \quad \varepsilon>0 .
$$


Obviously, multiplying (50) by $F^{\varepsilon}$ and integrating with respect to $(s, x, p)$ we control the $L^{2}$ norm of $F^{\varepsilon}(t, \cdot, \cdot, \cdot)$ uniformly in $t \in \mathbb{R}_{+}$and $\varepsilon>0$

$$
\left\|F^{\varepsilon}(t)\right\|^{2}=\left\|F^{\varepsilon}(0)\right\|^{2}=T\left\|f^{\text {in }}\right\|_{L^{2}\left(\mathbb{R}^{3} \times \mathbb{R}^{3}\right)}
$$

We intend to control the derivatives $\nabla_{(t, x, p)} F^{\varepsilon}$ as well, uniformly with respect to $\varepsilon>0$. The idea is to use the derivations commuting with $\mathcal{T}$, introduced in Proposition 4.6. Indeed, with the notation $a(t, s, x, p)=\left(0, \frac{p}{m}, e E(t, x)+\omega_{c} \theta(s){ }^{\perp} p\right)$ the equation (50) becomes

$$
\partial_{t} F^{\varepsilon}+a \cdot \nabla_{(s, x, p)} F^{\varepsilon}+\frac{1}{\varepsilon} \mathcal{T} F^{\varepsilon}=0 .
$$

Applying the operator $c^{i} \cdot \nabla_{(s, x, p)}, i \in\{1, \ldots, 6\}$ and taking into account that $c^{i}$. $\nabla_{(s, x, p)} \mathcal{T} F^{\varepsilon}=\mathcal{T}\left(c^{i} \cdot \nabla_{(s, x, p)} F^{\varepsilon}\right)$ one gets

$$
\partial_{t} G_{i}^{\varepsilon}+a \cdot \nabla_{(s, x, p)} G_{i}^{\varepsilon}+\left[c^{i}, a\right] \cdot \nabla_{(s, x, p)} F^{\varepsilon}+\frac{1}{\varepsilon} \mathcal{T} G_{i}^{\varepsilon}=0
$$

where $G_{i}^{\varepsilon}=c^{i} \cdot \nabla_{(s, x, p)} F^{\varepsilon}$ and $\left[c^{i}, a\right]$ are the Poisson brackets between the fields $c^{i}$, $i \in\{1, \ldots, 6\}$ and $a$. Multiplying $(56)$ by $G_{i}^{\varepsilon}$ and integrating with respect to $(s, x, p)$ yield

$$
\begin{aligned}
\frac{1}{2} \frac{d}{d t} \int_{0}^{T} \int_{\mathbb{R}^{3}} \int_{\mathbb{R}^{3}}\left|G_{i}^{\varepsilon}\right|^{2} \mathrm{~d} p \mathrm{~d} x \mathrm{~d} s & =-\int_{0}^{T} \int_{\mathbb{R}^{3}} \int_{\mathbb{R}^{3}} G_{i}^{\varepsilon}\left[c^{i}, a\right] \cdot \nabla_{(s, x, p)} F^{\varepsilon} \mathrm{d} p \mathrm{~d} x \mathrm{~d} s \\
& \leq\left\|G_{i}^{\varepsilon}(t)\right\|\left\|\left[c^{i}, a\right] \cdot \nabla_{(s, x, p)} F^{\varepsilon}(t)\right\|, \quad i \in\{1, \ldots, 6\}
\end{aligned}
$$

or equivalently

$$
\left\|G_{i}^{\varepsilon}(t)\right\| \leq\left\|G_{i}^{\varepsilon}(0)\right\|+\int_{0}^{t}\left\|\left[c^{i}, a\right] \cdot \nabla_{(s, x, p)} F^{\varepsilon}(\tau)\right\| \mathrm{d} \tau, \quad i \in\{1, \ldots, 6\} .
$$

It is easily seen that for any $i \in\{1, \ldots, 6\}$ the field $\left[c^{i}, a\right]$ has no component along $s$, since $c_{s}^{i}=a_{s}=0$. Therefore $\left[c^{i}, a\right] \in \operatorname{span}\left\{c^{1}, \ldots, c^{6}\right\}$

$$
\left[c^{i}, a\right]=\sum_{j=1}^{6} \gamma_{i j}(t, s, x, p) c^{j}, \quad i \in\{1, \ldots, 6\}
$$

for some coefficients $\gamma_{i j} \in L_{\text {loc }}^{1}\left(\mathbb{R}_{+} ; L^{\infty}\left(\mathbb{R} \times \mathbb{R}^{3} \times \mathbb{R}^{3}\right)\right), i, j \in\{1, \ldots, 6\}$. Actually we have

$$
\begin{gathered}
{\left[c^{1}, a\right]=-\frac{\omega_{c} \theta(s)}{2} c^{2}+\left(e \partial_{x_{1}} E_{1}-\frac{m \omega_{c}^{2}}{4} \theta^{2}(s)\right) c^{4}+e \partial_{x_{1}} E_{2} c^{5}+e \partial_{x_{1}} E_{3} c^{6}} \\
{\left[c^{2}, a\right]=\frac{\omega_{c} \theta(s)}{2} c^{1}+e \partial_{x_{2}} E_{1} c^{4}+\left(e \partial_{x_{2}} E_{2}-\frac{m \omega_{c}^{2}}{4} \theta^{2}(s)\right) c^{5}+e \partial_{x_{2}} E_{3} c^{6}}
\end{gathered}
$$


$\left[c^{3}, a\right]=\sum_{j=1}^{3} e \partial_{x_{3}} E_{j} c^{j+3}, \quad\left[c^{4}, a\right]=\frac{c^{1}}{m}-\frac{\omega_{c}}{2} \theta(s) c^{5}, \quad\left[c^{5}, a\right]=\frac{c^{2}}{m}+\frac{\omega_{c}}{2} \theta(s) c^{4}, \quad\left[c^{6}, a\right]=\frac{c^{3}}{m}$.

It follows that $\left[c^{i}, a\right] \cdot \nabla_{(s, x, p)} F^{\varepsilon}=\sum_{j=1}^{6} \gamma_{i j} G_{j}^{\varepsilon}$ and (57) yields

$$
\left\|G_{i}^{\varepsilon}(t)\right\| \leq\left\|G_{i}^{\varepsilon}(0)\right\|+\int_{0}^{t} \sum_{j=1}^{6}\left\|\gamma_{i j}(\tau)\right\|_{L^{\infty}}\left\|G_{j}^{\varepsilon}(\tau)\right\| \mathrm{d} \tau .
$$

We deduce that

$$
\sum_{i=1}^{6}\left\|G_{i}^{\varepsilon}(t)\right\| \leq \sum_{i=1}^{6}\left\|G_{i}^{\varepsilon}(0)\right\|+\int_{0}^{t} \gamma(\tau) \sum_{j=1}^{6}\left\|G_{j}^{\varepsilon}(\tau)\right\| \mathrm{d} \tau
$$

with $\gamma(\tau)=\max _{j \in\{1, \ldots, 6\}} \sum_{i=1}^{6}\left\|\gamma_{i j}(\tau)\right\|_{L^{\infty}}$ and by Gronwall's lemma we obtain that

$$
\left\{\nabla_{(x, p)} F^{\varepsilon}(t): t \in[0, I], \quad \varepsilon>0\right\}
$$

remains bounded in $L_{\#}^{2}\left(\mathbb{R}_{s} ; L^{2}\left(\mathbb{R}_{x}^{3} \times \mathbb{R}_{p}^{3}\right)\right)$. By Proposition 6.2 we know that $\cup_{\varepsilon>0, t \in[0, I]} \operatorname{supp} F^{\varepsilon}(t)$ remains into a compact set of $\mathbb{R}_{s} / T \mathbb{Z} \times \mathbb{R}^{3} \times \mathbb{R}^{3}$ and clearly there is a constant $C_{3}(I)$ such that for any $t \in[0, I]$

$$
\begin{aligned}
\sup _{\varepsilon>0}\left\|\frac{p}{m} \cdot \nabla_{x} F^{\varepsilon}(t)+\left(e E+\omega_{c} \theta(s){ }^{\perp} p\right) \cdot \nabla_{p} F^{\varepsilon}\right\| & \leq C_{3}(I)\left(1+\|E(t)\|_{L^{\infty}\left(\mathbb{R}^{3}\right)}\right) \\
& \leq C_{4}(I)\left(1+\|E(0)\|_{L^{\infty}\left(\mathbb{R}^{3}\right)}+\left\|\partial_{t} E\right\|_{L^{1}\left([0, t] ; L^{\infty}\left(\mathbb{R}^{3}\right)\right)}\right) .
\end{aligned}
$$

It remains to estimate the time derivative $\partial_{t} F^{\varepsilon}$. As before we write

$$
\partial_{t}\left(\partial_{t} F^{\varepsilon}\right)+a \cdot \nabla_{(s, x, p)}\left(\partial_{t} F^{\varepsilon}\right)+e \partial_{t} E \cdot \nabla_{p} F^{\varepsilon}+\frac{1}{\varepsilon} \mathcal{T}\left(\partial_{t} F^{\varepsilon}\right)=0
$$

implying that

$$
\begin{aligned}
\frac{1}{2} \frac{d}{d t}\left\|\partial_{t} F^{\varepsilon}\right\|^{2} & =-e \int_{0}^{T} \int_{\mathbb{R}^{3}} \int_{\mathbb{R}^{3}} \partial_{t} E(t) \cdot \nabla_{p} F^{\varepsilon}(t) \partial_{t} F^{\varepsilon}(t) \mathrm{d} p \mathrm{~d} x \mathrm{~d} s \\
& \leq\left\|e \partial_{t} E(t) \cdot \nabla_{p} F^{\varepsilon}(t)\right\|\left\|\partial_{t} F^{\varepsilon}(t)\right\| .
\end{aligned}
$$

We deduce that

$$
\left\|\partial_{t} F^{\varepsilon}(t)\right\| \leq\left\|\partial_{t} F^{\varepsilon}(0)\right\|+\int_{0}^{t}\left\|e \partial_{t} E(\tau)\right\|_{L^{\infty}}\left\|\nabla_{p} F^{\varepsilon}(\tau)\right\| \mathrm{d} \tau .
$$

The family of time derivatives $\left\{\partial_{t} F^{\varepsilon}(t): t \in[0, I], \varepsilon>0\right\}$ remains bounded in $L_{\#}^{2}\left(\mathbb{R}_{s} ; L^{2}\left(\mathbb{R}_{x}^{3} \times \mathbb{R}_{p}^{3}\right)\right)$ iff $\left\{\left\|\partial_{t} F^{\varepsilon}(0)\right\|: \varepsilon>0\right\}$ remains bounded in $L_{\#}^{2}\left(\mathbb{R}_{s} ; L^{2}\left(\mathbb{R}_{x}^{3} \times \mathbb{R}_{p}^{3}\right)\right)$. Notice that $\mathcal{T} F^{\varepsilon}(0)=0$ and therefore

$$
\begin{aligned}
\sup _{\varepsilon>0}\left\|\partial_{t} F^{\varepsilon}(0)\right\| & =\sup _{\varepsilon>0}\left\|-a \cdot \nabla_{(s, x, p)} F^{\varepsilon}(0)\right\| \\
& =\sup _{\varepsilon>0}\left\|-a \cdot \nabla_{(s, x, p)} f^{\text {in }}\left(x, p-\frac{m \omega_{c}}{2} \theta(s)^{\perp} x+\frac{m \omega_{c}}{2} \theta(0){ }^{\perp} x\right)\right\|<+\infty .
\end{aligned}
$$


Combining (58), (59) we deduce that there is a constant $C_{2}(I)$ such that

$$
\sup _{\varepsilon>0, t \in[0, I]}\left\|\left(\partial_{t}+\frac{p}{m} \cdot \nabla_{x}+\left(e E(t)+\omega_{c} \theta(s){ }^{\perp} p\right) \cdot \nabla_{p}\right) F^{\varepsilon}(t)\right\| \leq \varepsilon C_{2}(I)
$$

saying that $\sup _{\varepsilon>0, t \in[0, I]}\left\|\mathcal{T} F^{\varepsilon}(t)\right\| \leq \varepsilon C_{2}(I)$.

\section{$7 \quad$ Three dimensional setting}

In this section we study the particle dynamics under fast oscillating three dimensional magnetic fields

$$
B^{\varepsilon}(t, x)=\theta(t / \varepsilon) B(x) b(x), \operatorname{div}_{x}(B b)=0
$$

for some scalar positive function $B(x)$ and some field of unitary vectors $b(x) \in \mathbb{R}^{3}$. The analysis is completely analogous to that for fast oscillating homogeneous magnetic fields previously discussed. Therefore we only focus on the formal derivation of the limit model. By Gauss's magnetic law $\operatorname{div}_{x} B^{\varepsilon}=0$ we can write $B b=\operatorname{curl}_{x} A, \operatorname{div}_{x} A=0$ and by Faraday's law $\partial_{t} B^{\varepsilon}+\operatorname{curl}_{x} E^{\varepsilon}=0$ we deduce that the rotational part, $\operatorname{curl}_{x} \psi$, of the electric field $E^{\varepsilon}=-\nabla_{x} \phi+\operatorname{curl}_{x} \psi$ is given by

$$
\operatorname{curl}_{x} \psi=-\frac{1}{\varepsilon} \theta^{\prime}(t / \varepsilon) A(x) .
$$

The Vlasov equation becomes, with the notations $E=-\nabla_{x} \phi, \omega_{c}(x)=\frac{e B(x)}{m}$

$$
\partial_{t} f^{\varepsilon}+\frac{p}{m} \cdot \nabla_{x} f^{\varepsilon}+\left(e E(t, x)-\frac{e \theta^{\prime}(t / \varepsilon)}{\varepsilon} A(x)+\omega_{c}(x) \theta(t / \varepsilon) p \wedge b(x)\right) \cdot \nabla_{p} f^{\varepsilon}=0 .
$$

We prescribe the initial distribution

$$
f^{\varepsilon}(0, x, p)=f^{\text {in }}(x, p), \quad(x, p) \in \mathbb{R}^{3} \times \mathbb{R}^{3} .
$$

Plugging the Hilbert expansion (17) into (60) yields

$$
\partial_{s} f^{0}-e \theta^{\prime}(s) A(x) \cdot \nabla_{p} f^{0}=0
$$

at the lowest order $\varepsilon^{-1}$ and

$\partial_{t} f^{0}+\frac{p}{m} \cdot \nabla_{x} f^{0}+\left(e E(t, x)+\omega_{c}(x) \theta(s) p \wedge b(x)\right) \cdot \nabla_{p} f^{0}+\partial_{s} f^{1}-e \theta^{\prime}(s) A(x) \cdot \nabla_{p} f^{1}=0$ 
at the next order $\varepsilon^{0}$. As before, the point is how to eliminate the first order correction $f^{1}$ appearing in (63), based on the constraint (62). We introduce the operator

$$
\mathcal{T}_{1} u=\operatorname{div}_{(s, p)}\left\{u\left(1,-e \theta^{\prime}(s) A(x)\right)\right\}
$$

with domain

$D\left(\mathcal{T}_{1}\right)=\left\{u \in L_{\#}^{2}\left(\mathbb{R}_{s} ; L^{2}\left(\mathbb{R}_{x}^{3} \times \mathbb{R}_{p}^{3}\right)\right): \operatorname{div}_{(s, p)}\left\{u\left(1,-e \theta^{\prime}(s) A(x)\right)\right\} \in L_{\#}^{2}\left(\mathbb{R}_{s} ; L^{2}\left(\mathbb{R}_{x}^{3} \times \mathbb{R}_{p}^{3}\right)\right)\right\}$

The characteristics $(S, X, P)(\tau ; s, x, p)$ of the first order differential operator $\mathcal{T}_{1}$ are given by

$$
S(\tau ; s, x, p)=s+\tau, \quad X(\tau ; s, x, p)=x, \quad P(\tau ; s, x, p)=p+e(\theta(s)-\theta(s+\tau)) A(x) .
$$

Notice that a complete family of functional independent invariants is given by $\{x, p+$ $e \theta(s) A(x)\}$ and therefore the constraint (62) becomes

$$
\exists g^{0}=g^{0}(t, x, q): f^{0}(t, s, x, p)=g^{0}(t, x, q=p+e \theta(s) A(x)) .
$$

In particular $f^{0}(t, t / \varepsilon, x, p)$ is fast oscillating through the periodic profile $\theta(s=t / \varepsilon)$ and therefore we expect that $f^{0}(t, s, x, p)$ is the two-scale limit of $f^{\varepsilon}(t, x, p)$ when $\varepsilon \searrow 0$. The average operator $\langle\cdot\rangle_{1}$ along the characteristic flow (64) is given by

$$
\begin{aligned}
\langle u\rangle_{1}(s, x, p) & =\frac{1}{T} \int_{0}^{T} u(S(\tau ; s, x, p), X(\tau ; s, x, p), P(\tau ; s, x, p)) \mathrm{d} \tau \\
& =\frac{1}{T} \int_{0}^{T} u(s+\tau, x, p+e(\theta(s)-\theta(s+\tau)) A(x)) \mathrm{d} \tau \\
& =\frac{1}{T} \int_{0}^{T} u(\tau, x, p+e \theta(s) A(x)-e \theta(\tau) A(x)) \mathrm{d} \tau
\end{aligned}
$$

for any function $u \in L_{\#}^{2}\left(\mathbb{R}_{s} ; L^{2}\left(\mathbb{R}_{x}^{3} \times \mathbb{R}_{p}^{3}\right)\right)$. The dynamics for $f^{0}$ is obtained by eliminating $f^{1}$ in (63) taking into account that the functions in the range of $\mathcal{T}_{1}$ are zero average. We have

$\partial_{t} f^{0}+\frac{p}{m} \cdot \nabla_{x} f^{0}+\left(e E(t, x)+\omega_{c}(x) \theta(s) p \wedge b(x)\right) \cdot \nabla_{p} f^{0}=-\mathcal{T}_{1} f^{1} \in$ Range $\mathcal{T}_{1}=\operatorname{ker}\langle\cdot\rangle_{1}$ and therefore (63) is equivalent to

$$
\left\langle\partial_{t} f^{0}+\frac{p}{m} \cdot \nabla_{x} f^{0}+\left(e E(t, x)+\omega_{c}(x) \theta(s) p \wedge b(x)\right) \cdot \nabla_{p} f^{0}\right\rangle_{1}=0
$$

We need to average the derivatives with respect to $(t, x, p)$ of the density $f^{0}$, under the constraint (62). Clearly we have $\left\langle\partial_{t} f^{0}\right\rangle_{1}=\partial_{t}\left\langle f^{0}\right\rangle_{1}=\partial_{t} f^{0}$. 
Lemma 7.1 Assume that $f(s, x, p)=g(x, q=p+e \theta(s) A(x))$ is smooth. Then we have

$$
\begin{aligned}
& \left\langle\frac{p}{m} \cdot \nabla_{x} f\right\rangle_{1}=\frac{q-e\langle\theta\rangle A(x)}{m} \cdot \nabla_{x} g+\frac{e}{m} \partial_{x} A\left(\langle\theta\rangle q-e\left\langle\theta^{2}\right\rangle A\right) \cdot \nabla_{q} g \\
= & \frac{p+e(\theta(s)-\langle\theta\rangle) A(x)}{m} \cdot \nabla_{x} f+\frac{e}{m} \partial_{x} A\left((\langle\theta\rangle-\theta) p+e\left(2 \theta\langle\theta\rangle-\theta^{2}-\left\langle\theta^{2}\right\rangle\right) A(x)\right) \cdot \nabla_{p} f
\end{aligned}
$$

and

$$
\begin{aligned}
\left\langle\left(e E(x)+\omega_{c} \theta p \wedge b\right) \cdot \nabla_{p} f\right\rangle_{1} & =\left[e E+\omega_{c}\left(\langle\theta\rangle q \wedge b-e\left\langle\theta^{2}\right\rangle A(x) \wedge b\right)\right] \cdot \nabla_{q} g \\
& =\left[e E+\omega_{c}\langle\theta\rangle p \wedge b+\omega_{c} e\left(\theta\langle\theta\rangle-\left\langle\theta^{2}\right\rangle\right) A(x) \wedge b\right] \cdot \nabla_{p} f .
\end{aligned}
$$

Proof. We have

$$
\nabla_{x} f=\nabla_{x} g+e \theta(s){ }^{t} \partial_{x} A \nabla_{q} g, \quad \nabla_{p} f=\nabla_{q} g
$$

Since $\nabla_{(x, q)} g$ are constant along the flow (64) we can write

$$
\left\langle\frac{p}{m} \cdot \nabla_{x} f\right\rangle_{1}=\frac{\langle p\rangle_{1}}{m} \cdot \nabla_{x} g+\frac{e}{m}\langle p \theta\rangle_{1} \cdot{ }^{t} \partial_{x} A \nabla_{q} g .
$$

It is easily seen by the definition of the average operator $\langle\cdot\rangle_{1}$ that

$$
\langle p\rangle_{1}=p+e \theta(s) A(x)-e\langle\theta\rangle A(x)
$$

and

$$
\langle p \theta\rangle_{1}=(p+e \theta(s) A(x))\langle\theta\rangle-e\left\langle\theta^{2}\right\rangle A(x)
$$

implying that

$$
\left\langle\frac{p}{m} \cdot \nabla_{x} f\right\rangle_{1}=\frac{q-e\langle\theta\rangle A(x)}{m} \cdot \nabla_{x} g+\frac{e}{m} \partial_{x} A\left(\langle\theta\rangle q-e\left\langle\theta^{2}\right\rangle A(x)\right) \cdot \nabla_{q} g .
$$

Similarly one gets

$$
\left\langle\left(e E(x)+\omega_{c} \theta p \wedge b\right) \cdot \nabla_{p} f\right\rangle_{1}=\left[e E+\omega_{c}\left(\langle\theta\rangle q \wedge b-e\left\langle\theta^{2}\right\rangle A(x) \wedge b\right)\right] \cdot \nabla_{q} g
$$

Combining the previous computations and using the identities

$$
\partial_{x} A A+A \wedge \operatorname{curl}_{x} A={ }^{t} \partial_{x} A A, \quad \partial_{x} A q+q \wedge \operatorname{curl}_{x} A={ }^{t} \partial_{x} A q
$$


yield the transport equation in the space phase $(x, q)$

$$
\partial_{t} g^{0}+\frac{q-e\langle\theta\rangle A(x)}{m} \cdot \nabla_{x} g^{0}+\left(e E+\frac{e}{m}\langle\theta\rangle^{t} \partial_{x} A q-\frac{e^{2}}{m}\left\langle\theta^{2}\right\rangle{ }^{t} \partial_{x} A A\right) \cdot \nabla_{q} g^{0}=0
$$

where $f^{0}(t, s, x, p)=g^{0}(t, x, q=p+e \theta(s) A(x))$, since $\mathcal{T}_{1} f^{0}(t)=0$ for any $t \in \mathbb{R}_{+}$. The transport equation in the phase space $(x, p)$ becomes

$$
\begin{aligned}
\partial_{t} f^{0} & +\frac{p+e(\theta(s)-\langle\theta\rangle) A(x)}{m} \cdot \nabla_{x} f^{0}+\left[e E+\frac{e}{m}\left(\langle\theta\rangle{ }^{t} \partial_{x} A-\theta \partial_{x} A\right) p\right. \\
& \left.+\frac{e^{2}}{m}\left(\theta\langle\theta\rangle-\left\langle\theta^{2}\right\rangle\right){ }^{t} \partial_{x} A A+\frac{e^{2}}{m}(\langle\theta\rangle-\theta) \theta \partial_{x} A A\right] \cdot \nabla_{p} f^{0}=0 .
\end{aligned}
$$

We supplement these transport equations by the initial conditions

$$
\begin{gathered}
g^{0}(0, x, q)=f^{\text {in }}(x, q-e \theta(0) A(x)) \\
f^{0}(0, s, x, p)=f^{\text {in }}(x, p+e(\theta(s)-\theta(0)) A(x)) .
\end{gathered}
$$

Following the lines in Sections 5, 6 we can prove weak and strong convergence results, which justify the Hilbert expansion in (17). In the weak framework we obtain

Theorem 7.1 Assume that $E \in L_{\text {loc }}^{1}\left(\mathbb{R}_{+} ; L^{\infty}\left(\mathbb{R}^{3}\right)\right), A \in L_{\text {loc }}^{1}\left(\mathbb{R}_{+} ; W^{1, \infty}\left(\mathbb{R}^{3}\right)\right)^{3}, f^{\text {in }} \in$ $L^{2}\left(\mathbb{R}^{3} \times \mathbb{R}^{3}\right)$. For any $\varepsilon>0$ let $f^{\varepsilon} \in L^{\infty}\left(\mathbb{R}_{+} ; L^{2}\left(\mathbb{R}^{3} \times \mathbb{R}^{3}\right)\right)$ be a weak solution of $(60)$, (61). Then there is a sequence $\varepsilon_{n} \searrow 0$ such that $\left(f^{\varepsilon_{n}}\right)_{n}$ two-scale converges towards a weak solution of (67), (69).

\section{References}

[1] G. Allaire, Homogenization and two-scale convergence, SIAM J. Math. Anal. 23(1992) 1482-1518.

[2] V.I. Arnold, Ecuaţii diferenţiale ordinare, Editura Ştiinţifica şi Enciclopedica, Bucureşti, 1978.

[3] N.N. Bogoliubov, Y.A. Mitropolsky, Asymptotic methods in the theory of nonlinear oscillations, Gordon and Breach Sciences Publishers, New York, 1961.

[4] M. Bostan, The Vlasov-Poisson system with strong external magnetic field. Finite Larmor radius regime, Asymptot. Anal., 61(2009) 91-123. 
[5] M. Bostan, The Vlasov-Maxwell system with strong initial magnetic field. Guiding-center approximation, SIAM J. Multiscale Model. Simul. 6(2007) 10261058.

[6] M. Bostan, Transport equations with singular coefficients. Application to the gyrokinetic models in plasma physics, research report INRIA, hal:inria-00232800, submitted 2009.

[7] M. Bostan, Gyrokinetic Vlasov equation in three dimensional setting. Second order approximation, hal-00431289, submitted 2009.

[8] Y. Brenier, Convergence of the Vlasov-Poisson system to the incompressible Euler equations, Comm. Partial Differential Equations 25(2000) 737-754.

[9] A.J. Brizard, T.S. Hahm, Foundations of nonlinear gyrokinetic theory, Rev. Modern Phys., 79(2007) 421-468.

[10] E. Frénod, E. Sonnendrücker, Homogenization of the Vlasov equation and of the Vlasov-Poisson system with strong external magnetic field, Asymptotic Anal. 18(1998) 193-213.

[11] E. Frénod, E. Sonnendrücker, The finite Larmor radius approximation, SIAM J. Math. Anal. 32(2001) 1227-1247.

[12] F. Golse, L. Saint-Raymond, The Vlasov-Poisson system with strong magnetic field, J. Math. Pures Appl. 78(1999) 791-817.

[13] F. Golse, L. Saint-Raymond, The Vlasov-Poisson system with strong magnetic field in quasineutral regime, Math. Models Methods Appl. Sci. 13(2003) 661-714.

[14] V. Grandgirard, M. Brunetti, P. Bertrand, N. Besse, X. Garbet, P. Ghendrih, G. Manfredi, Y. Sarazin, O. Sauter, E. Sonnendrücker, J. Vaclavik, L. Villard, A drift-kinetic semi-Lagrangian 4D code for ion turbulence simulation, J. Comput. Phys. 217(2006) 395-423.

[15] R.D. Hazeltine, J.D. Meiss, Plasma confinement, Dover Publications, Inc. Mineola, New York, 2003. 
[16] P. Morel, E. Gravier, N. Besse, A. Ghizzo, P. Bertrand, The water bag model and gyrokinetic applications, Commun. Nonlinear Sci. Numer. Simul. 13(2008) $11-17$.

[17] G. N'Guetseng, A general convergence result for a functional related to the theory of homogenization, SIAM J. Math. Anal. 20(1989) 608-623.

[18] M. Reed, B. Simon, Methods of Modern Mathematical Physics, Vol. I, Functional Analysis, Academic Press 1980. 